\title{
YAP activity protects against endotoxemic acute lung injury by activating multiple mechanisms
}

\author{
LING-YAN LIU ${ }^{1,2^{*}}$, XIAO-QIONG SHAN ${ }^{1,3^{*}}$, FU-KUN ZHANG $^{1}$, XIAO-FANG FAN ${ }^{1}$, JUN-MING FAN ${ }^{1}$, \\ YONG-YU WANG ${ }^{1}$, SHU FANG LIU ${ }^{1}$, SUN-ZHONG MAO ${ }^{1}$ and YONG-SHENG GONG ${ }^{1}$ \\ ${ }^{1}$ Institute of Hypoxia Medicine, School of Basic Medical Sciences, Wenzhou Medical University, Wenzhou, \\ Zhejiang 325035; ${ }^{2}$ Department of Occupational Diseases, Zhejiang Quhua Hospital, Quzhou, Zhejiang 324004; \\ ${ }^{3}$ Department of Pathology, Hangzhou Third People's Hospital, Hangzhou, Zhejiang 310009, P.R. China
}

Received April 17, 2020; Accepted September 2, 2020

DOI: $10.3892 / \mathrm{ijmm} .2020 .4759$

\begin{abstract}
The roles of the Hippo-Yes-associated protein (YAP) pathway in lung injury and repair remain elusive. The present study examined the effects of systemic inhibition or stimulation of YAP activity on lung injury, repair and inflammation in a mouse model of lipopolysaccharide (LPS)-induced lung injury. Mice were treated with or without YAP inhibitor, verteporfin, or with or without YAP stimulator, XMU-MP-1, and intraperitoneally injected with LPS $(7.5 \mathrm{mg} / \mathrm{kg})$. Lung injury and repair were evaluated by histological analysis and by testing for markers of lung injury. Lung inflammation was assessed by measuring tissue levels of inflammatory mediators. Lung injury was associated with a decreased, whereas lung repair was associated with an increased YAP activity evidenced by nuclear translocation. Lung injury was associated with a high level of lung inflammation and epithelial adherens junction disassembly, but not with cell proliferation or epithelial cell regeneration. The injury phase was defined as $0-48 \mathrm{~h}$ post-LPS injection, and the $48-168 \mathrm{~h}$ time period was considered the repair phase. Inhibition of YAP activity at the injury phase, using verteporfin, exacerbated, whereas its stimulation, using XMU-MP-1, alleviated lung injury, lung inflammation and epithelial adherens junction disassembly. Inhibition or stimulation of YAP activity at the injury phase had no effects on cell proliferation or epithelial regeneration. By contrast, lung repair was associated with inflammation resolution, increased cell proliferation, epithelial regeneration
\end{abstract}

Correspondence to: Professor Sun-Zhong Mao or Professor Yong-Sheng Gong, Institute of Hypoxia Medicine, School of Basic Medical Sciences, Wenzhou Medical University, Room 7A211, Tongren Building, University Town, Chashan, Wenzhou, Zhejiang 325035, P.R. China

E-mail:wzlmao@126.com

E-mail: fxbwzmc@126.com

*Contributed equally

Key words: lung injury and repair, Hippo-Yes-associated protein pathway, epithelial adherens junction, inflammation, acute lung injury, regeneration, epithelial cells and reassembly of epithelial adherens junctions. Inhibition of YAP activity at the repair phase delayed inflammation resolution, impeded lung recovery, inhibited cell proliferation and epithelial regeneration, and inhibited epithelial adherens junction reassembly. Stimulation of YAP activity at the repair phase reversed all these processes. The results of the current study demonstrated that the Hippo-YAP activity serves a protective role against endotoxemic lung injury. The Hippo-YAP activity alleviated lung inflammation and injury at the injury phase and promoted inflammation resolution and lung repair at the repair phase.

\section{Introduction}

Acute lung injury (ALI) or acute respiratory distress syndrome (ARDS) is a clinical condition that imposes a substantial health burden (1-6). A prospective epidemiologic study estimated an annual incidence of ALI/ARDS of 190,000 adult patients in the United States with an associated 74,500 deaths per year (3-6). Despite progress in the understanding of the molecular and cellular mechanisms of ALI/ARDS, and significant advances in supporting therapies, ALI/ARDS-related mortality rate remains high (1-6). ALI/ARDS is characterized by inflammation, disruption of endothelial and epithelial barrier integrity, and tissue injury in the lungs, resulting in lung interstitial edema and leakage of proteins and immune cells into the alveolar space (1-3). Accelerating lung repair, and promoting the resolution of lung inflammation and edema is a potential therapeutic strategy for ALI/ARDS $(2,3)$. It is, therefore, essential to understand the mechanisms and signaling pathways that regulate these processes. However, the mechanisms and pathways underlying the regulation of lung repair and inflammation resolution are poorly understood $(2,3,6)$.

The Hippo-YAP pathway is an evolutionarily conserved cell survival signaling complex that controls tissue growth and organ size (7-11). Key components of this signaling pathway include mammalian Ste20-like kinases 1/2 (Mst1/2), large tumor suppressor kinases 1/2 (LATS1/2) and downstream effectors, including Yes-associated protein (YAP) and transcriptional coactivator with PDZ-binding motif (TAZ) (7-11). YAP and TAZ are transcriptional coactivators that regulate gene expression by interacting with TEA/ATTS domain 
transcription factor 1-4 (TEAD1-4) (12). YAP/TAZ activity is regulated by their phosphorylation and subcellular translocation (7-11). When the Hippo pathway is activated, Mst1/2 phosphorylate and activate LATS1/2.LATS1/2 kinases, in turn, phosphorylate YAP and TAZ and cause them to be transported out of the nucleus, leading to the inhibition of their activity and thus, to the inhibition of expression of their target genes (7-11). When the Hippo pathway is inactivated, Mst1/2 and LATS1/2 activities are inhibited, and YAP/TAZ are dephosphorylated and translocated into the nucleus where they interact with the TEADs to induce the expression of their target genes (7-12).

The Hippo-YAP pathway plays a regulatory role in organ development and lung regeneration after pneumonectomy. Hippo-YAP integrates with upstream growth signals $(13,14)$ to stimulate cell proliferation, differentiation and migration (15-17), promote stem and progenitor cell self-renewal, lineage commitment and expansion (18-21), and stimulate angiogenesis (22), leading to organogenesis, tissue growth and regeneration. Moreover, Hippo-YAP senses physical and geographic cues in growing tissue and thus mediates tissue-derived feedback signals that counteract the upstream growth signals $(15,16,23-26)$. The reciprocal regulation of upstream growth signals and tissue-derived feedback signals mediated by the Hippo-YAP pathway ensures a balance between tissue growth and maturation (27) and prevents organ overgrowth $(8,9)$.

Although the Hippo-YAP pathway is known to regulate multiple biological processes that play important roles in organ development and tissue growth (7-22), there have been few studies examining the roles of Hippo-YAP activity in organ repair and organ function recovery after injury. YAP activity has been reported to mediate peripheral nerve repair after injury (28), and to regulate hepatic repair following ischemia reperfusion liver injury (29). The roles of the Hippo-YAP pathway in lung injury and repair are just beginning to be studied. Compared to cells with normal level of LATS2 expression, mesenchymal stem cells with downregulated LATS2 expression are more effective in protecting against lipopolysaccharide (LPS)-induced ALI (30). Naphthalene-mediated lung injury causes nuclear translocation of YAP, which then stimulates expansion of basal stem/progenitor cells to promote regeneration (31). LPS- or Streptococcus pneumoniae-induced lung injury is associated with increased YAP/TAZ nuclear translocation $(32,33)$. Selective knockdown of YAP or TAZ in alveolar type II epithelial cells (AECII) impairs epithelial regeneration in mouse models of pneumonia (32). However, new epithelial cell generation is only one step in the process of epithelial barrier repair. The newly generated epithelial cells must be incorporated into the epithelial layer and form cell-cell junctions with their neighboring cells on the epithelial layer to restore epithelial barrier function (34-36). It is unclear whether the Hippo-YAP pathway regulates interepithelial junction formation. Moreover, lung repair involves multiple components, including repair of the endothelial barrier, restoration of lung structure, and resolution of lung inflammation and edema $(3,34,35,37,38)$. To the best of our knowledge, whether and how the Hippo-YAP pathway may regulate these biological processes is not known. It is also unclear whether the Hippo-YAP pathway modulates lung injury. Thus, the roles of this pathway in inflammatory lung injury and repair remain to be elucidated.
In the current study, YAP activity was systemically inhibited or stimulated to examine the effects of the Hippo-YAP pathway on lung injury and repair in a mouse model of LPS-induced ALI/ARDS. The results of the present study demonstrated that the Hippo-YAP activity protected against lung injury by activating multiple mechanisms.

\section{Materials and methods}

Animal studies. Adult male ICR mice (age, 8-10 weeks) were supplied by Shanghai SLAC Laboratory Animal Co., Ltd. Mice were housed in a temperature-controlled room $\left(22 \pm 2^{\circ} \mathrm{C}\right)$ with 12-h light/dark cycle and relative humidity of $40-60 \%$. Mice had free access to food and water. A total of 320 mice (25-30 g) were used for the studies. All animal experiments were approved by the Institutional Animal Care and Use Committee of Wenzhou Medical University (Wenzhou, China) and were performed in accordance with the Guide for the Care and Use of Laboratory Animals of the National Institute of Health (39). For a dose-response study, mice (n=30/group) were injected with equal volume of saline or $5,7.5$ or $10 \mathrm{mg} / \mathrm{kg}$ Escherichia coli LPS [cat. no. 0111:B4; intraperitoneal injection (i.p.); Sigma-Aldrich; Merck KGaA]. The survival rate was monitored for 7 days. Mice were closely observed to identify animals that have reached humane endpoints and required euthanasia. Those endpoints included slow, rapid, labored or agonal breathing, cardiac failure, impaired ambulation, inability to obtain food/water, ruffled fur, severe lethargy and signs of severe distress. Those mice were euthanized and counted as non-surviving animals in the survival rate analysis.

For time course studies, mice were injected with an equal volume of saline or Escherichia coli LPS (7.5 mg/kg; i.p.), and lung inflammation and injury, cell proliferation and YAP activity were assessed at $0,6,12,24,48,72,120$ and $168 \mathrm{~h}$ post-LPS injection ( $\mathrm{n}=5 /$ time-point). Mice were anesthetized with pentobarbital sodium $(50 \mathrm{mg} / \mathrm{kg}$; i.p.; cat. no. $4579 / 50$; R\&D Systems, Inc.). At the end of each experiment, mice were euthanized by $\mathrm{CO}_{2}$ asphyxia. Mice were put into a euthanasia chamber. $\mathrm{CO}_{2}$ was allowed to flow into the chamber at a displacement rate of $40 \%$ chamber volume/minute until mice were no longer breathing. Death was confirmed by loss of vital signs, including heartbeat and breathing. Euthanasia was performed when mice have reached humane endpoints for survival study, and at the end of each experiment for other studies.

The effects of inhibiting YAP activity were studies at 24 , 48, 72 and $168 \mathrm{~h}$ post-LPS injection. There were four study groups for each timepoint, control, verteporfin (VP), LPS and LPS + VP groups (3 mice per group for biochemical analyses; 5 mice per group for functional studies). Mice in control and LPS groups were injected with $0.1 \%$ DMSO (solvent for VP), and mice in VP and LPS + VP groups were injected with VP $(100 \mathrm{mg} / \mathrm{kg}$, i.p.; AdooQ Bioscience). Mice in $24 \mathrm{~h}$ groups were injected with a single dose of VP $(100 \mathrm{mg} / \mathrm{kg}) 2 \mathrm{~h}$ before LPS injection. Mice in 48, 72 and $168 \mathrm{~h}$ groups were initially injected with VP $(100 \mathrm{mg} / / \mathrm{kg})$ at $20 \mathrm{~h}$ after LPS injection, followed by an additional $100 \mathrm{mg} / \mathrm{kg}$ injection every $24 \mathrm{~h}$. Mice in control and VP groups were injected with equal volume of saline, and mice in LPS and LPS + VP groups were injected with LPS (7.5 mg/kg; i.p.). At 24, 48, 72 and $168 \mathrm{~h}$ 
after LPS or saline injection, bronchoalveolar lavage (BAL) was performed, and lung inflammation and injury, cell proliferation and YAP activity were assessed.

The effects of stimulating YAP activity were studies at 24 , 48, 72 and $168 \mathrm{~h}$ post-LPS injection. There were four study groups for each timepoint, control, XMU-MP-1, LPS and LPS + XMU-MP-1 groups (3 mice per group for biochemical analyses; 5 mice per group for functional studies). Mice in control and LPS groups were injected with $0.1 \%$ citric acid containing 20\% Kolliphor HS (solvent for XMU-MP-1; cat. no. 42966; Sigma-Aldrich; Merck KGaA), and mice in XMU-MP-1 and LPS + XMU-MP-1 groups were injected with XMU-MP-1 (1 mg/kg; i.p.). Mice in $24 \mathrm{~h}$ groups were injected with a single dose of XMU-MP-1 (1 mg/kg) $2 \mathrm{~h}$ before LPS injection. Mice in 48, 72 and $168 \mathrm{~h}$ groups were initially injected with XMU-MP-1 (1 mg/kg) at $20 \mathrm{~h}$ after LPS injection, followed by an additional dose $(1 \mathrm{mg} / \mathrm{kg})$ injection every $24 \mathrm{~h}$. Mice in control and XMU-MP-1 groups were injected with saline, and mice in LPS and LPS+ XMU-MP-1 groups were injected with LPS (7.5 mg/kg, i.p.). At 24, 48, 72 or $168 \mathrm{~h}$ after saline or LPS injection, BAL was performed, and lung inflammation and injury, cell proliferation and YAP activity were assessed. XMU-MP-1 was synthesized as previously described (40).

Histological examination. Lungs were perfused with PBS under deep anaesthesia, fixed with $4 \%$ paraformaldehyde at $4^{\circ} \mathrm{C}$ for $24 \mathrm{~h}$, processed by passing through increasing concentrations of ethanol and paraffin embedded, or embedded in optimal cutting temperature compound (cat. no. 4583; Beijing Solarbio Science \& Technology Co., Ltd.) and snap frozen at $-80^{\circ} \mathrm{C}$ for immunofluorescence staining, as described below. For histological analysis, paraffin-embedded sections (6- $\mu \mathrm{m}$ thick) were stained with hematoxylin and eosin at room temperature for 5-10 min. Stained sections were examined under a light microscope (Nikon Eclipse 80i; Nikon Corporation) at a magnification of $\times 200$.

BAL and analysis. BAL was performed by delivering $0.8 \mathrm{ml}$ of pre-warmed EDTA-saline into trachea followed by gentle chest massage and suction. Following removal of debris, BAL fluid (BALF) from three BALs was pooled and centrifuged at $4^{\circ} \mathrm{C}$ at $1,650 \mathrm{x}$ for $3 \mathrm{~min}$. The pellet and supernatant were collected.

BALF concentration of TNF- $\alpha$ (cat. no. JL10484-96T) and IL-6 (cat. no. JL20268-96T) was quantified using ELISA kits (J\&L Biological, Inc.). BALF protein concentration, as an indicator of epithelial permeability, was determined using a BCA protein assay kit (Thermo Fisher Scientific, Inc.). To measure myeloperoxidase (MPO) activity, as an indicator of neutrophil infiltration, the cell pellets were homogenized in $5 \mathrm{mM}$ phosphate buffer and then centrifuged at $15,000 \times \mathrm{g}$ at $4^{\circ} \mathrm{C}$ for $20 \mathrm{~min}$. The pellets were resuspended in phosphate buffer containing $0.5 \%$ hexadecyltrimethylammonium bromide, subjected to a cycle of freezing at $-80^{\circ} \mathrm{C}$ for $1 \mathrm{~h}$ and thawing at $4^{\circ} \mathrm{C}$ for $1 \mathrm{~h}$, and assayed for MPO activity (MPO assay kit; cat. no. A0441-1; Nanjing Jiancheng Bio-Engineering Institute). MPO activity was measured by continuously recording absorbance at $460 \mathrm{~nm}$ for $3 \mathrm{~min}$. MPO activity was presented as activity unit/l (U/1).
Reverse transcription quantitative-PCR (RT-qPCR). RNA was isolated from lungs using RNAiso Plus kit (Takara Bio, Inc.) and reverse transcribed into cDNA using a two-step cDNA synthesis kit (Takara Bio, Inc.) according to the manufacturer's protocols. qPCR was performed using SYBR-Green PCR master mix (Takara Bio, Inc.) and an ABI Prism 7900 sequence detection system (Applied Biosystems; Thermo Fisher Scientific, Inc). The following thermocycling conditions were used: Initial denaturation at $95^{\circ} \mathrm{C}$ for $15 \mathrm{~min}$; 40 cycles at $95^{\circ} \mathrm{C}$ for $10 \mathrm{sec}, 60^{\circ} \mathrm{C}$ for $20 \mathrm{sec}$ and $72^{\circ} \mathrm{C}$ for $32 \mathrm{sec}$. The primer sequences are included in Table I. Results were analyzed using the $2^{-\Delta \Delta \mathrm{Cq}}$ method (41). The mRNA level of each target gene was normalized to peptidylprolyl isomerase B (PPIB) level.

Western blotting. Total, cytoplasmic or nuclear protein fractions were extracted from lungs, as previously described $(42,43)$. Equal amounts of proteins (30 $\mu \mathrm{g} / \mathrm{lane}$ ) were separated using SDS-PAGE (10\% gel) and transferred to polyvinylidene fluoride membranes (EMD Millipore). Membranes were blocked with 5\% non-fat dry milk in TBST $(10 \mathrm{mM} / 1$ Tris- $\mathrm{HCl}, \mathrm{pH} 7.5$; $150 \mathrm{mM} / 1 \mathrm{NaCl} ; 0.1 \%$ Tween-20) at room temperature for $1 \mathrm{~h}$, and incubated overnight at $4^{\circ} \mathrm{C}$ with antibodies against YAP (1:1,000; cat. no. 4912S; Cell Signaling Technology, Inc.), phosphorylated (p)-YAP (1:1,000; cat. no. 4912S; Cell Signaling Technology, Inc.), connective tissue growth factor (CTGF; 1:500; cat. no. sc-101586; Santa Cruz Biotechnology, Inc.), proliferating cell nuclear antigen (PCNA; 1:500; cat. no. ab29; Abcam), pulmonary surfactant apoprotein C (Sp-c; 1:1,000; cat. no. ab90716; Abcam), aquaporin 5 (AQP-5; 1:1,000; cat. no. ab78486; Abcam), E-cadherin (1:4,000, cat. no. 20874-1-AP; Proteintech Group, Inc.), GADPH (1:5,000, cat. no. AB2302; EMD Millipore), $\beta$-actin (1:1,000; cat. no. WH100959; ABclonal Biotech Co., Ltd.) and type B1 nuclear lamin (lamin B1; 1:5,000; cat. no. ab16048; Abcam). The membranes were washed, incubated at room temperature for $1.5 \mathrm{~h}$ with horseradish peroxidase-conjugated secondary antibody (anti-mouse/rabbit IgG; 1:3,000; cat. nos. A2304 and A0545; Sigma-Aldrich; Merck KGaA), and washed again. Immunoreactive bands were visualized using an enhanced chemiluminescent reagent (Bio-Rad laboratories, Inc.). The blot band intensities were semi-quantified using ImageJ version 1.46 (National Institutes of Health).

Immunofluorescence (IF) staining. Cryosections (6- $\mu \mathrm{m}$ thick) were fixed with $4 \%$ paraformaldehyde at $4{ }^{\circ} \mathrm{C}$ for $15 \mathrm{~min}$, permeabilized with $0.1 \%$ Triton X-100 (cat. no. T8200; Beijing Solarbio Science \& Technology Co., Ltd., https://solarbio. en.alibaba.com) at $4^{\circ} \mathrm{C}$ for $10 \mathrm{~min}$, blocked with $5 \%$ BSA (cat. no. A3858; Sigma-Aldrich; Merck KGaA) at $4^{\circ} \mathrm{C}$ for $15 \mathrm{~min}$ and stained with anti-AQP-5 (1;200, cat. no. ab78486; Abcam), anti-podoplanin (PDPN, 1;200, cat. no. ab11936; Abcam), anti-Sp-c (1:100, cat. no. sc-7706; Santa Cruz, Biotechnology, Inc.) antibodies at $4^{\circ} \mathrm{C}$ for $4 \mathrm{~h}$, followed by washing and staining with Alexa Fluor 594-conjugated goat anti-rabbit (1:500, cat. no. 33112ES60; Shanghai Yeasen Biotechnology Co., Ltd.), donkey anti-rabbit (1:500, cat. no. 34212ES60; Shanghai Yeasen Biotechnology Co., Ltd.) or goat anti-hamster IgG (1:500, cat. no. A-21113; Invitrogen; Thermo Fisher Scientific, Inc.) at room temperature for $1 \mathrm{~h}$. These sections were washed, 
Table I. Primer sequences used for quantitative PCR.

Primer sequence (5'-3')

\begin{tabular}{lll}
\cline { 2 - 3 } Target & \multicolumn{1}{c}{ Forward } & \multicolumn{1}{c}{ Reverse } \\
\hline Sp-c & ATGGACATGAGTAGCAAAGAGGT & CACGATGAGAAGGCGTTTGAG \\
CTGF & CTCCACCCGAGTTACCAATG & TGGCGATTTTAGGTGTCCG \\
CYR61 & GGAGGTGGAGTTAACGAGAAAC & GTGGTCTGAACGATGCATTTC \\
PDPN & ACCGTGCCAGTGTTGTTCTG & AGCACCTGTGGTTGTTATTTTGT \\
Yap & ACGACTTCCTCAACAGTGTG & TCATTGCATCTCCTTCCAGTG \\
E-cad & CAGGTCTCCTCATGGCTTTGC & CTTCCGAAAAGAAGGCTGTCC \\
IL-1 $\beta$ & GCAACTGTTCCTGAACTCAACT & ATCTTTTGGGGTCCGTCAACT \\
IL-6 $\alpha$ & TAGTCCTTCCTACCCCAATTTCC & TTGGTCCTTAGCCACTCCTTC \\
TNF- $\alpha$ & CCCTCACACTCAGATCATCTTCT & GCTACGACGTGGGCTACAG \\
IL-10 & GCTCTTACTGACTGGCATGAG & CGCAGCTCTAGGAGCATGTG \\
Cyclin A2 & GCCTTCACCATTCATGTGGAT & TTGCTGCGGGTAAAGAGACAG \\
Ki-67 & ATCATTGACCGCTCCTTTAGGT & GCTCGCCTTGATGGTTCCT \\
PPIB & GGCTCCGTCGTCTTCCTTTT & ACTCGTCCTACAGATTCATCTCC
\end{tabular}

CTGF, connective tissue growth factor; CYR61, cellular communication network factor 1; PDPN, podoplanin; Sp-c, pulmonary surfactant apoprotein C; Yap, Yes-associated protein; E-cad, E-cadherin; PPIB, peptidylprolyl isomerase B.

blocked again with $5 \% \mathrm{BSA}$ at $4^{\circ} \mathrm{C}$ for $15 \mathrm{~min}$ and stained with anti-Ki-67 (1:25; cat. no. AF7649; R\&D Systems, Inc.) or anti-YAP (1:50, cat. no. 4912S; Cell Signaling Technology, Inc.) antibodies at $4^{\circ} \mathrm{C}$ overnight, followed by washing and staining with Alexa Fluor 488-conjugated rabbit anti-goat (1:500; cat. no. 33706ES60), donkey anti-rabbit (1:500; cat. no.34206ES60) or FITC-conjugated rabbit anti-sheep IgG (1:500; cat. no. 33807ES60) (all Shanghai Yeasen Biotechnology Co., Ltd.) at room temperature for $1 \mathrm{~h}$. The slides were washed, nuclei counterstained with DAPI (Sigma-Aldrich; Merck KGaA) at room temperature for $15 \mathrm{~min}$, and mounted with mounting medium (Beyotime Institute of Biotechnology). Slides were viewed under a Zeiss confocal microscope (ZeissLSM800; Carl Zeiss AG), and images were captured and analyzed using Zeiss imagine analyzing system (ZEISS ZEN lite 2011; Carl Zeiss AG).

Assessment of endothelial permeability. Microvascular endothelial permeability was assessed using a dual albumin tracer method. A total of $2 \mathrm{~h}$ prior to sacrifice, mice in control, 24, 48, 72, 120 and $168 \mathrm{~h}$ groups (total 18 mice, 3 mice per group) were anesthetized and injected with $100 \mu \mathrm{g}$ mouse FITC-labeled BSA (FITC-BSA; cat. no. R-H-10026; Xi'an Qiyue Biotechnology, Co.) and then injected with $100 \mu \mathrm{g}$ mouse TRITC-labeled BSA (TRITC-BSA, cat. no. R-H-10085; Xi'an Qiyue Biotechnology, Co.) 2 h later via the jugular vein. Two minutes after TRITC-BSA injection, blood $(0.2 \mathrm{ml})$ was withdrawn, and lungs were excised and weighed. Lungs were homogenized, centrifuged at $500 \mathrm{x} \mathrm{g}$ at $4^{\circ} \mathrm{C}$ for $5 \mathrm{~min}$ and supernatant was prepared. FITC-BSA and TRITC-BSA fluorescence intensity in each supernatant and plasma sample was read using a Varioskan Flash multimode reader (Thermo Fisher Scientific, Inc.). Endothelial permeability was assessed based on extravascular leakage of FITC-BSA, expressed as FITC to TRITC fluorescence ratio.
Statistical analysis. Data are presented as the mean \pm SEM. GraphPad Prism 5.0 (GraphPad Software, Inc.) was used to perform statistical analyses. Comparisons between multiple groups with one and two factors were made using one-way and two-way ANOVA. Post hoc analyses were performed using Bonferroni post hoc test. Comparison between two groups was made using two-sided unpaired Student's t-test. $\mathrm{P}<0.05$ was considered to indicate a statistically significant difference.

\section{Results}

Time course profile of lung injury and repair in LPS-induced $A L I$. The repair process after lung injury can last days, depending on the cause of the injury $(37,38)$. One major challenge when studying lung repair mechanisms is to develop an animal model that produces significant lung injury, and yet has sufficient survival rate so that the process and mechanisms of lung repair can be studied in detail $(37,38)$. For this purpose, a dose-response study was initially performed (Fig. 1B). Injecting mice with $7.5 \mathrm{mg} / \mathrm{kg}$ LPS produced marked lung injury (Fig. 1A) and resulted in significant inflammation compared with the control group (Fig. 1C-F) but resulted in a 1-week survival rate of $\sim 65 \%$ (Fig. 1B). This LPS dose was chosen for subsequent experiments.

The entire course of lung injury and recovery after the LPS challenge was followed. The major pathological features of ALI/ARDS, inflammation, tissue injury, and endothelial and epithelial permeability changes were followed. Histological examination of lungs from control, 6, 12, 24, 48, 72, 120 and $168 \mathrm{~h}$ groups of mice showed that LPS caused marked lung tissue injury, as evidenced by inflammatory cell infiltration, hemorrhage, increased alveolar wall thickness and alveolar distortion. Lung injury deteriorated progressively between 6 to $48 \mathrm{~h}$, peaked at $48 \mathrm{~h}$, and then recovered gradually between 
A LPSOh
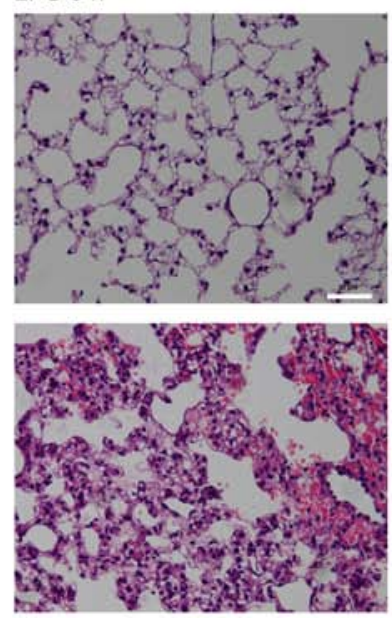

$48 \mathrm{~h}$
$6 \mathrm{~h}$
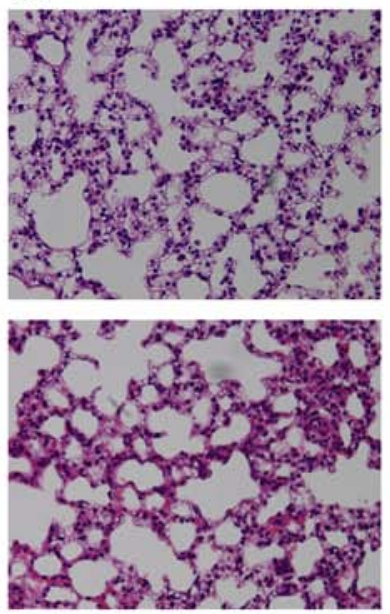

$72 \mathrm{~h}$
$12 \mathrm{~h}$
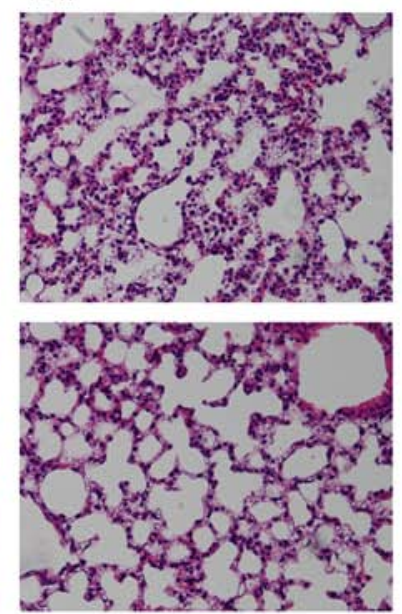

$120 \mathrm{~h}$
$24 \mathrm{~h}$
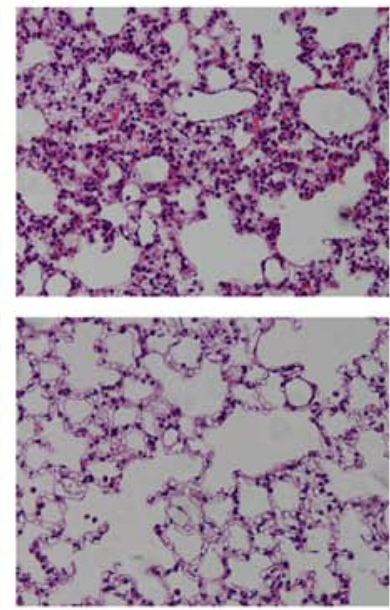

$168 \mathrm{~h}$
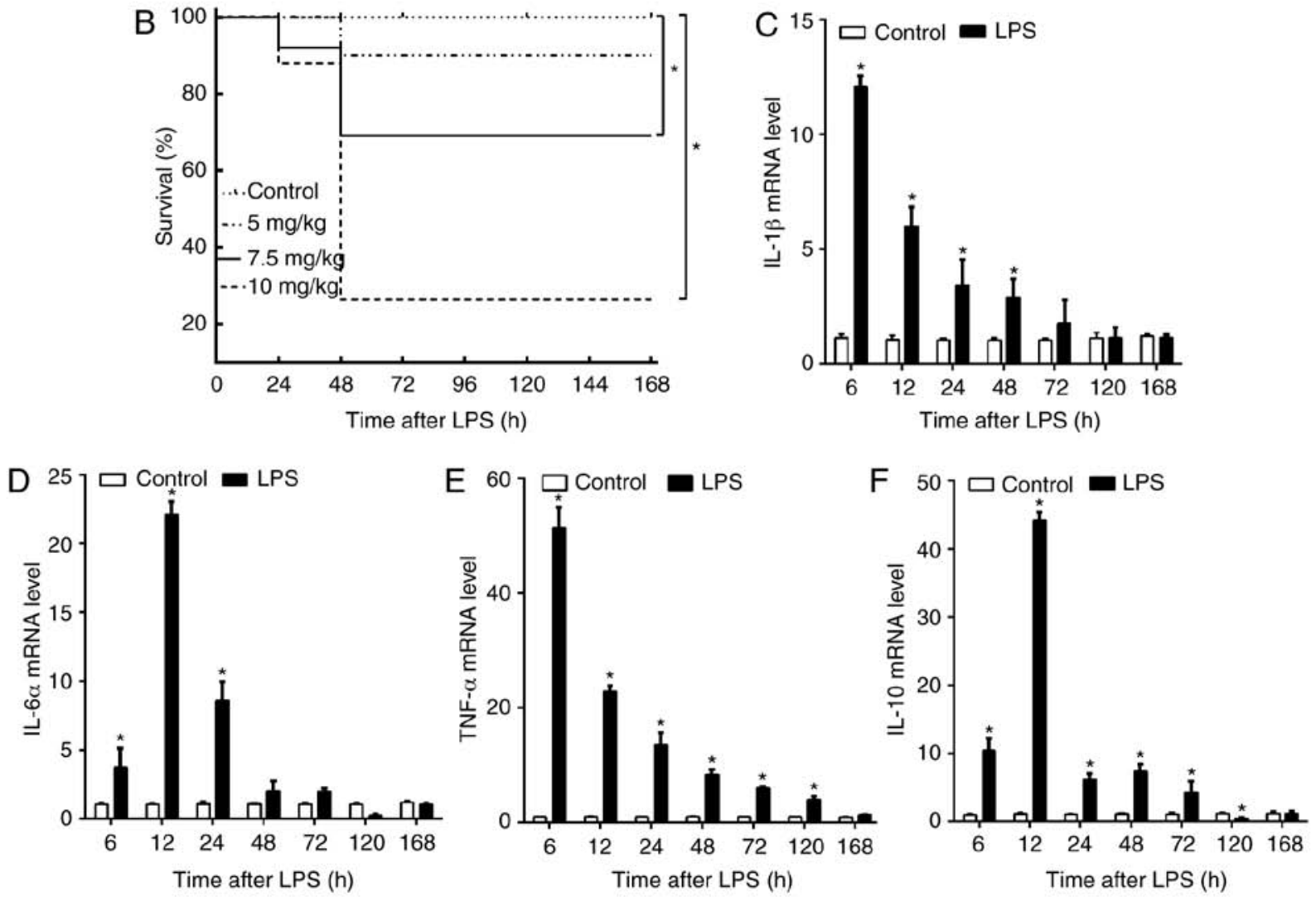

Figure 1. Time course of lung injury and recovery in LPS-induced acute lung injury. (A) Histological examination of lung injury in mice at $0,6,12,24$, 48, 72, 120 and $168 \mathrm{~h}$ post-LPS injection. Similar result were obtained in all 5 mice at each respective time-point. Magnification, x200. (B) Dose-response studies showed 1-week survival rate after injections with different doses of LPS. * $\mathrm{P}<0.05$ compared with the control group ( $\mathrm{n}=30$ mice/group). Reverse transcription-quantitative PCR analysis of lung tissue mRNA expression levels of (C) IL-1 $\beta$, (D) IL-6 $\alpha$, (E) TNF- $\alpha$ and (F) IL-10. Data are presented as the mean \pm SEM ( $n=3$ mice/group; 2 experimental repeats). ${ }^{*} \mathrm{P}<0.05$ vs. the corresponding control group. LPS, lipopolysaccharide.

48 to $168 \mathrm{~h}$ to reach full recovery at $168 \mathrm{~h}$ post-LPS injection (Fig. 1A). Lung inflammation, as assessed by tissue mRNA expression levels of inflammatory and anti-inflammatory cytokines, peaked at 6-12 $\mathrm{h}$ and decreased rapidly after that to reach a low level within $48 \mathrm{~h}$ post-LPS injection (Fig. 1C-F). The low level of lung inflammation persisted until $120 \mathrm{~h}$, and was not observed at $168 \mathrm{~h}$ post-LPS injection (Fig. 1C-F). Changes in lung epithelial permeability followed a similar time course to lung tissue injury and recovery, with damage peaking at $48 \mathrm{~h}$, followed by gradual recovery between 48 and $168 \mathrm{~h}$ post-LPS injection (Figs. 1A and 2B). An increase in lung endothelial permeability followed a slightly different time course, peaking at $24 \mathrm{~h}$, remained high till $48 \mathrm{~h}$, and then decreased rapidly between 72 and $120 \mathrm{~h}$, to finally reach the control level at $168 \mathrm{~h}$ post-LPS injection (Figs. 1A and 2A).

Lung injury and repair are associated with decreased and increased YAP activity, respectively. Based on the aforementioned time course profile, $0-48 \mathrm{~h}$ post-LPS injection was considered as the injury phase and $48-168 \mathrm{~h}$ as the repair 

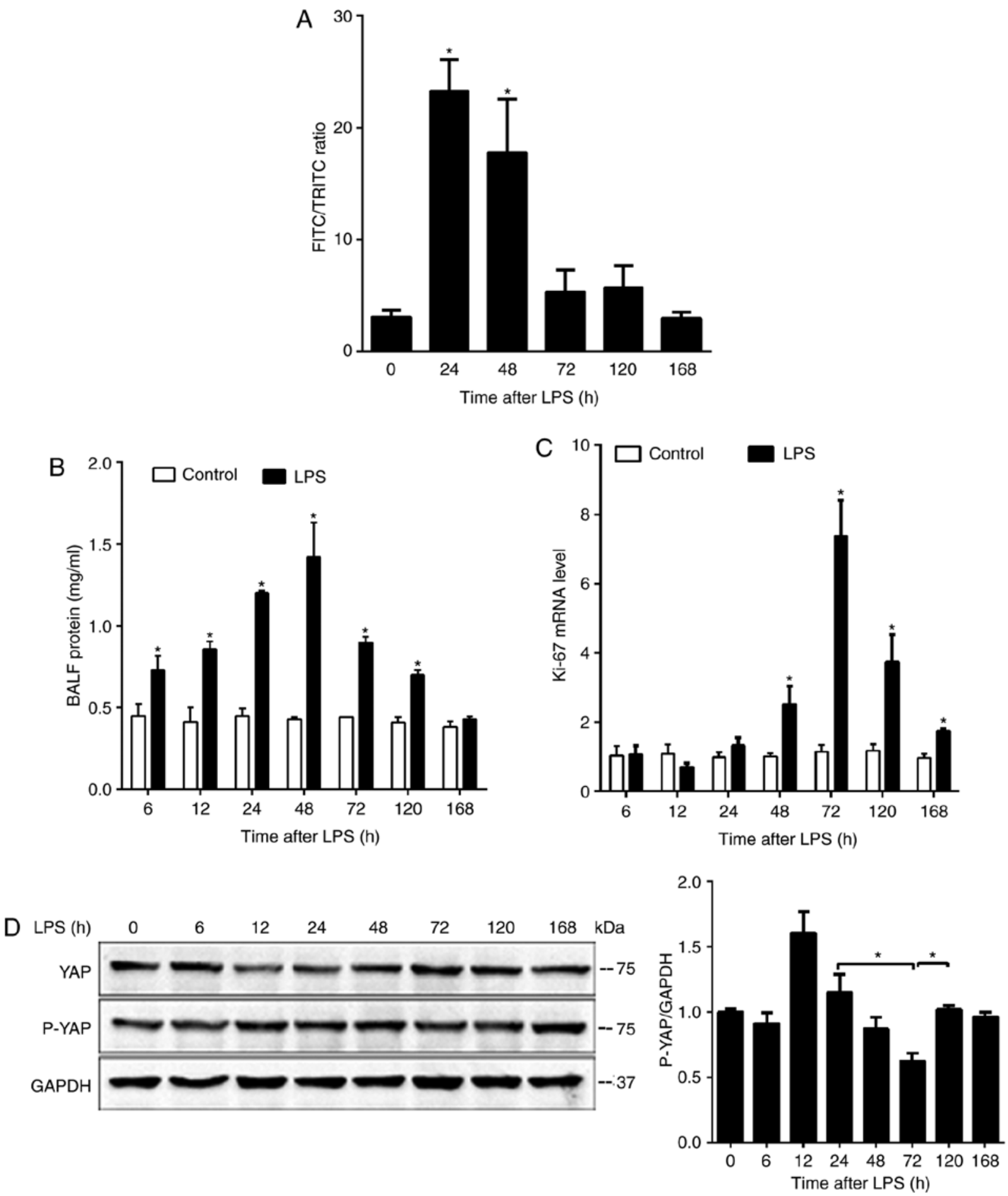

Figure 2. Lung injury and recovery are associated with a decreased or increased YAP activity, respectively. Mice were intraperitoneally injected with saline or $7.5 \mathrm{mg} / \mathrm{kg} \mathrm{LPS}$, and endothelial and epithelial permeability, lung tissue expression levels of Ki-67 and YAP phosphorylation levels were measured at the indicated time-points. (A) Endothelial permeability measured using FITC- and TRITC-labeled BSA. (B) Epithelial permeability estimated by measuring BALF protein concentration. (C) Reverse transcription-quantitative PCR analysis of lung tissue mRNA expression level of proliferation marker Ki-67. (D) Lung tissue p-YAP/YAP ratio determined using western blotting. Data are presented as the mean \pm SEM ( $n=3$ mice/group). "P $<0.05$ vs. the corresponding control group. BALF, bronchoalveolar lavage fluid; p, phosphorylated; YAP, Yes-associated protein; LPS, lipopolysaccharide.

phase. Within these phases, 6-24 $\mathrm{h}$ was termed the progressive injury phase, and $72-120 \mathrm{~h}$ was termed the active repair phase. This definition is consistent with the time course of cell proliferation (Fig. 2C), a major mechanism of lung repair. The tissue mRNA expression level of the proliferation marker $\mathrm{Ki}-67$ remained stable at the $6-24 \mathrm{~h}$ progressive injury phase, increased at the $48 \mathrm{~h}$ post-LPS injection transition phase, markedly increased at the $72-120 \mathrm{~h}$ active repair phase and decreased to near the control level at $168 \mathrm{~h}$ fully recovery (Fig. 2C). Whether lung injury and recovery were associated with alterations in YAP activity was also determined. YAP activity is controlled by its phosphorylation which reduces YAP nuclear translocation and transactivation activity (5-9). The p-YAP/YAP ratio in the lung tissue was significantly 

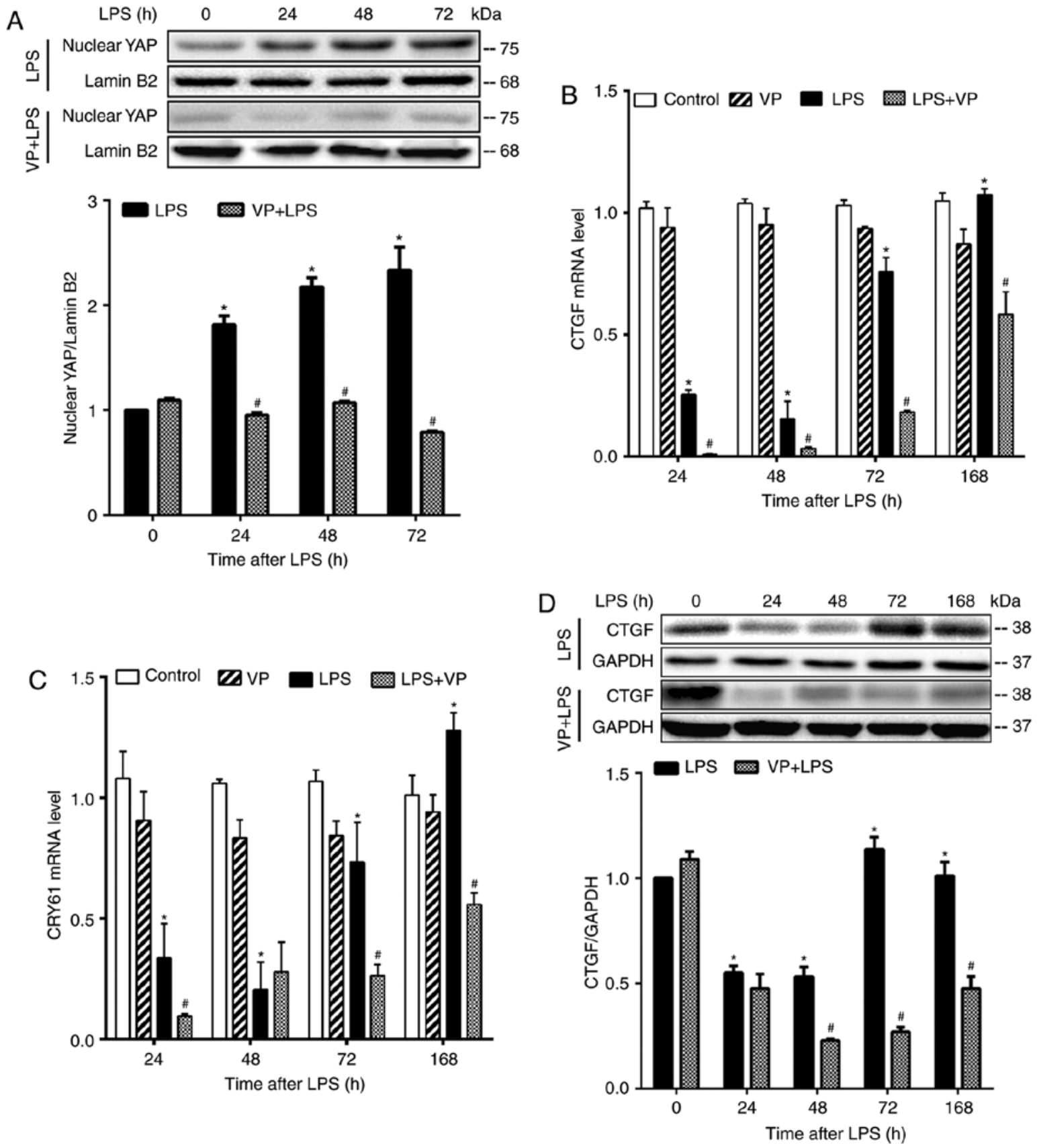

Figure 3. Verteporfin inhibits YAP activity. Mice were intraperitoneally injected with DMSO (solvent for VP), VP (100 mg/kg), LPS or LPS + VP. Lung tissue level of YAP activity was assessed at the indicated time-points. (A) Western blot analysis of nuclear YAP protein expression levels. Reverse transcription-quantitative PCR analysis of mRNA expression levels of (B) CTGF and (C) CYR61. (D) Western blot analysis of CTGF protein expression levels in the lungs. Data are presented as the mean \pm SEM ( $n=3$ mice/group; 2 experimental repeats/animals). ${ }^{*} \mathrm{P}<0.05$ vs. the control group; and ${ }^{\#} \mathrm{P}<0.05$ vs. the LPS alone group. VP, verteporfin; CTGF, connective tissue growth factor; CYR61, cysteine-rich angiogenic inducer 61; LPS, lipopolysaccharide; YAP, Yes-associated protein.

increased at $12 \mathrm{~h}$ (progressive injury phase) and significantly decreased at $72 \mathrm{~h}$ (active repair phase) (Fig. 2D), suggesting that lung injury and repair are associated with a decreased or increased YAP activity, respectively (Fig. 2).

Inhibition of YAP activity exacerbates lung injury and impedes lung recovery. To understand the roles of YAP activity in lung injury and repair, LPS-challenged mice were treated with VP, a small molecule that suppresses YAP activity by binding to it, and thereby preventing the formation of the TEAD-YAP complex in the nucleus (44). Treatment of mice with VP markedly reduced nuclear YAP protein content in
LPS-treated lungs, but not in control lungs (Fig. 3A), and inhibited the mRNA expression of CTGF and cellular communication network factor 1 (CYR61), two YAP target genes, in LPS-treated lungs (Fig. 3B and C). Compared to LPS alone group, VP also inhibited CTGF protein expression (Fig. 3D). These results confirmed that VP suppressed LPS-stimulated YAP activity in the lungs.

The effects of YAP activity inhibition on lung inflammation and lung injury and recovery were subsequently examined at different pathological stages. Compared with the LPS alone group, mice in the LPS + VP group displayed more severe tissue injury (Fig. 4A), significantly higher tissue expression 
A
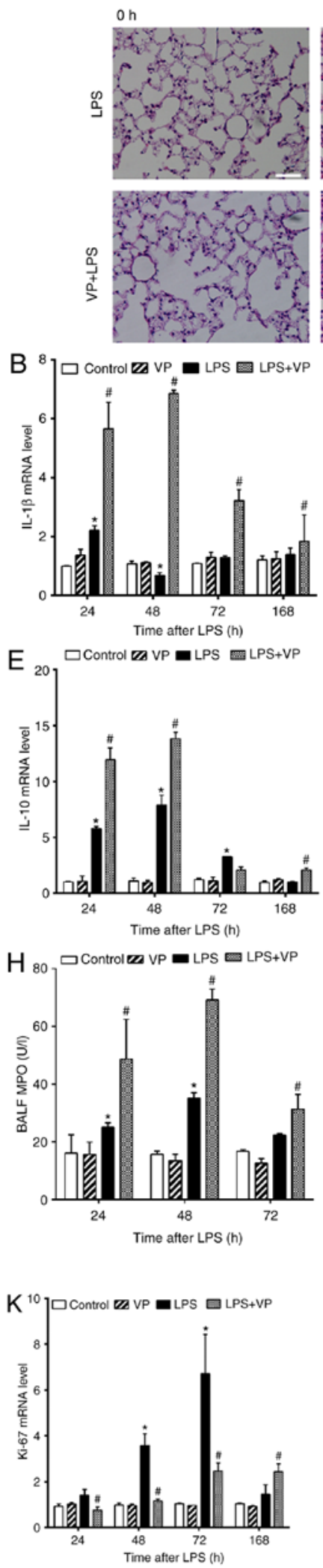

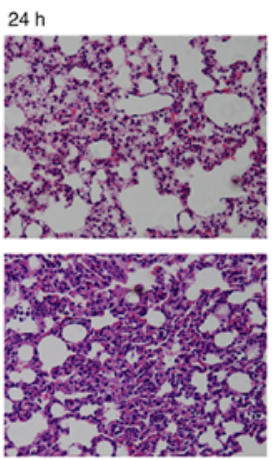

$48 \mathrm{~h}$

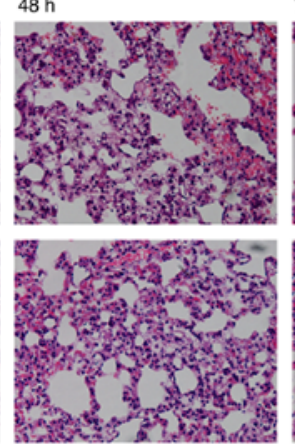

$72 \mathrm{~h}$
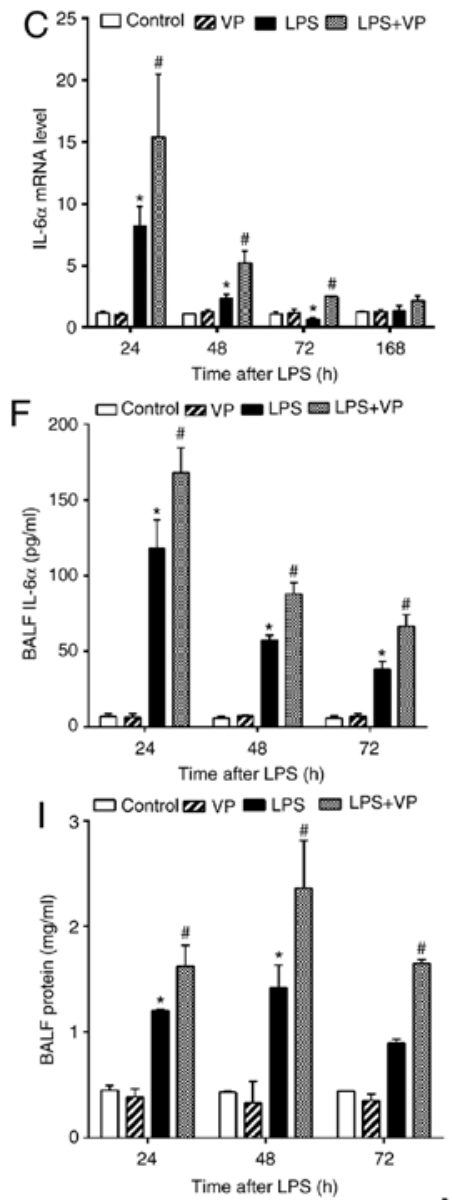

M

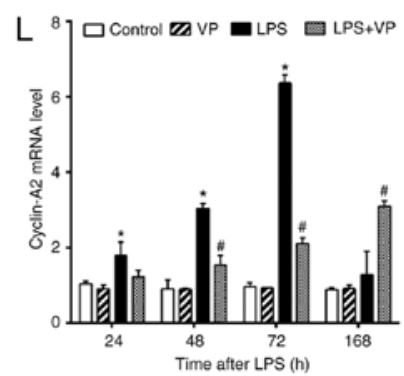

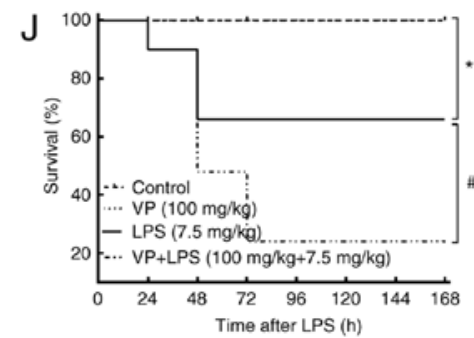
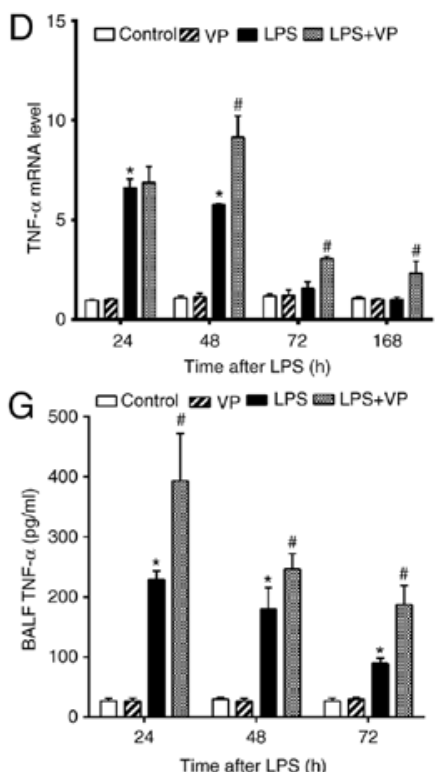

Time after LPS (h)
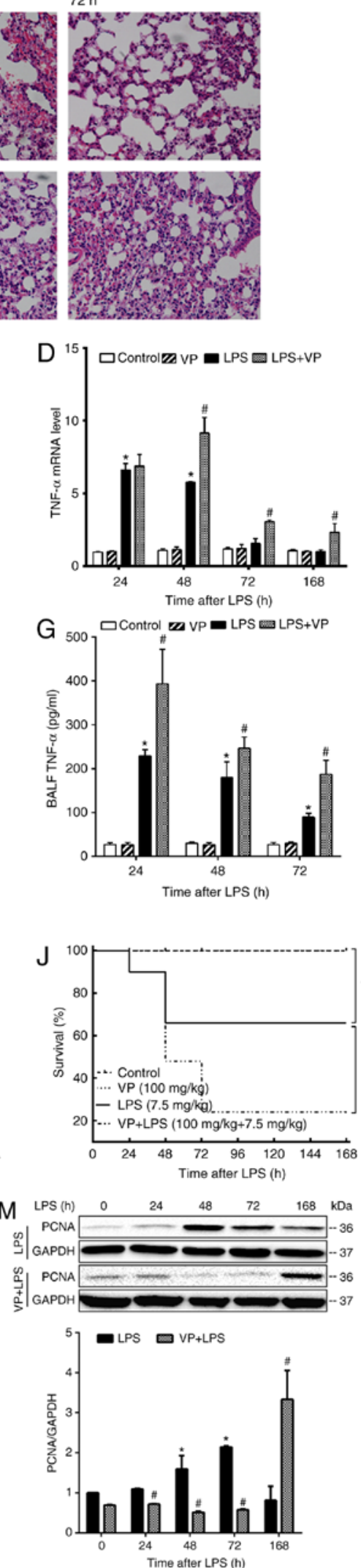

Figure 4. Continued. Inhibition of YAP activity exacerbates lung injury and impedes lung recovery. Mice were intraperitoneally injected with DMSO (solvent for VP), VP (100 mg/kg), LPS or LPS + VP. The effects of inhibiting YAP activity on lung injury and recovery, and on lung inflammation were evaluated at injury and repair phases. (A) Histological examination of lung injury. Similar result were obtained in all 5 mice at each respective time-point. Magnification, x200. Reverse transcription-quantitative PCR analysis of tissue expression levels of (B) IL-1 $\beta$, (C) IL-6 $\alpha$, (D) TNF- $\alpha$ and (E) IL-10. Data are presented as the mean \pm SEM ( $n=3$ mice per group; 2 experimental repeats/animal). Analyses of BALF levels of cytokines, including (F) IL- $6 \alpha$ and (G) TNF- $\alpha,(H)$ MPO activity, and (I) total protein concentration. Data are presented as the mean \pm SEM ( $\mathrm{n}=5$ mice/group). (J) Inhibition of YAP activity significantly reduced the survival rate ( $\mathrm{n}=30$ mice/group). Analyses of tissue expression levels of $(\mathrm{K}) \mathrm{Ki}-67$ and (L) cyclin-A2 mRNA, and (M) PCNA protein. Data are presented as the mean \pm SEM ( $n=3$ mice/group; 2 experimental repeats/animal). ${ }^{*} \mathrm{P}<0.05$ vs. the control group and ${ }^{~} \mathrm{P}<0.05$ vs. the LPS alone group. LPS, lipopolysaccharide; YAP, Yes-associated protein; BALF, bronchoalveolar lavage fluid; MPO, myeloperoxidase; PCNA, proliferating cell nuclear antigen; VP, verteporfin. 

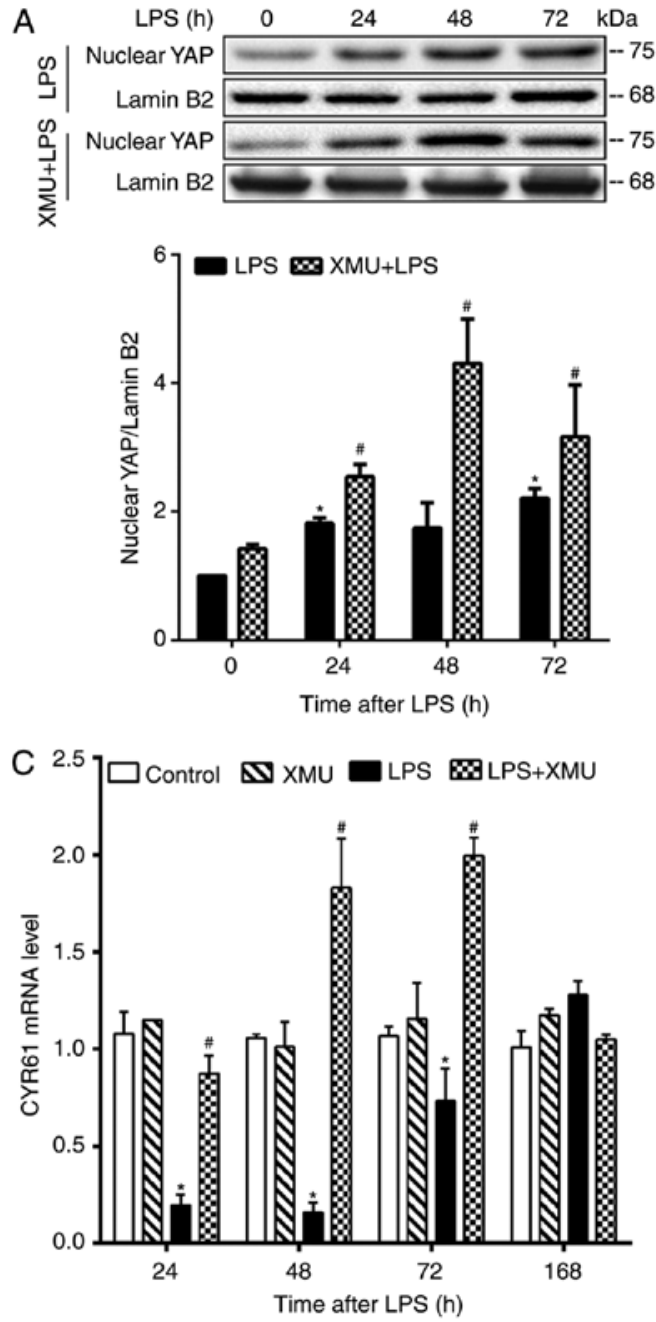
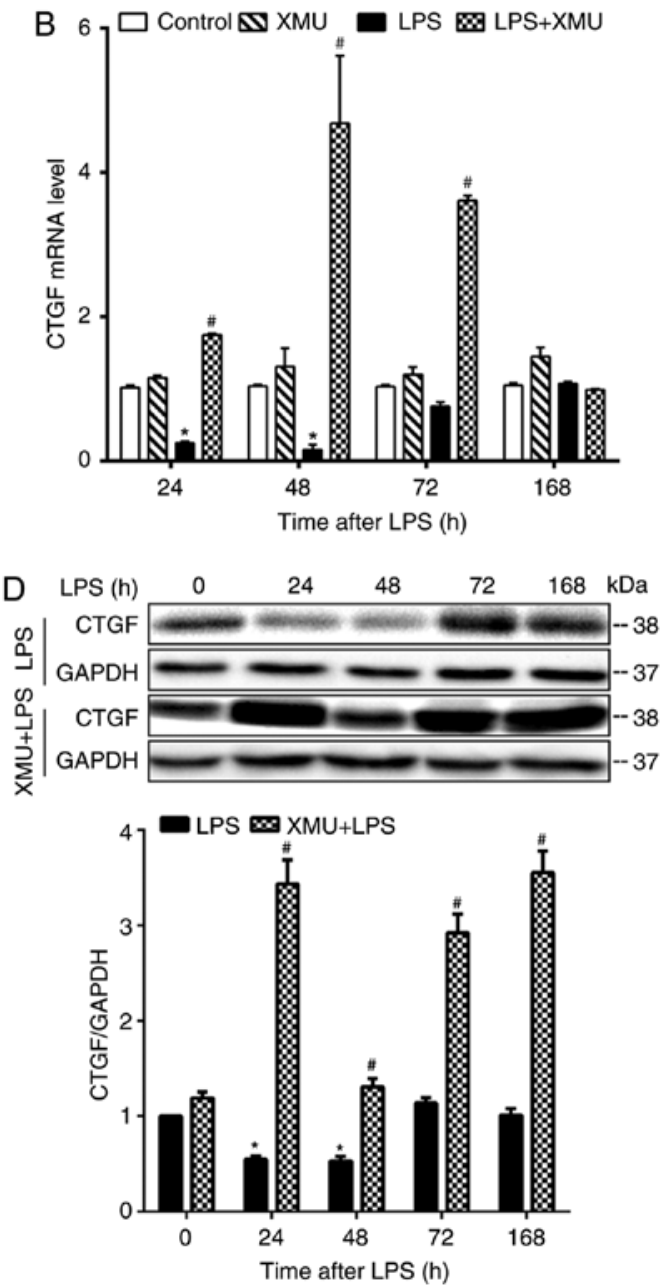

Figure 5. XMU-MP-1 stimulates YAP activity. Mice were injected intraperitoneally with $0.1 \%$ citric acid containing 20\% Kolliphor HS (solvent for XMU-MP-1), $1 \mathrm{mg} / \mathrm{kg}$ XMU-MP-1, LPS or LPS + XMU-MP-1. Lung tissue level of YAP activity was assessed at the indicated time-points. (A) Western blot analysis of the nuclear content of YAP protein. Reverse transcription-quantitative PCR analysis of (B) CTGF and (C) CYR61 mRNA expression levels. (D) Western blot analysis of CTGF protein expression levels in the lungs. Data are presented as the mean \pm SEM ( $\mathrm{n}=3$ mice/group; 2 experimental repeats/animal). "P<0.05 vs. the control group; and " $\mathrm{P}<0.05$ vs. the LPS alone group. XMU, XMU-MP-1; LPS, lipopolysaccharide; YAP, Yes-associated protein; CTGF, connective tissue growth factor; CYR61, cellular communication network factor 1.

levels of IL-1 $\beta$, IL- $6 \alpha$, TNF- $\alpha$ and IL-10 (Fig. 4B-E), significantly elevated BALF levels of IL- $6 \alpha$ and TNF- $\alpha$ (Fig. 4F-G), significantly increased BALF MPO activity (Fig. 4H), and significantly increased BALF protein content indicating higher epithelial permeability (Fig. 4I) at all time-points examined. Exacerbated lung inflammation and injury caused by VP treatment led to a worse outcome. Compared with mice in LPS alone group, mice in the LPS + VP group showed a delayed return to the control level in inflammatory cytokine expression (Fig. 4B-E) and delayed recovery from the injury (Fig. 4A and F-I). The 1-week survival rate has decreased from $76 \%$ in the LPS group to $48 \%$ in the LPS + VP group (Fig. 4J).

Inhibition of YAP activity in VP-treated mice significantly decreased tissue expression levels of cell proliferation markers, including Ki-67, cyclin-A2 and PCNA (Fig. 4K-M). YAP inhibition by VP appeared to have different effects on the expression of cell proliferation markers at different timepoints. Compared with mice in the LPS alone group, mice treated with LPS + VP had significantly reduced expression levels of $\mathrm{Ki}-67$ and PCNA at 24, 48 and $72 \mathrm{~h}$, and a reduced level of cyclin-A 2 at 48 and $72 \mathrm{~h}$ post LPS injection (Fig. 4K-M). By contrast, mice in the LPS + VP group had significantly increased levels of Ki-67, PCNA and cyclin-A2 expression at $168 \mathrm{~h}$ post LPS injection (Fig. 4K-M).

Stimulation of YAP activity attenuates lung injury and promotes lung recovery. YAP activity was stimulated by treating control and LPS-challenged mice with XMU-MP-1. The effects of this stimulation on lung inflammation, injury and recovery were examined at different pathological stages. XMU-MP-1 is a selective Mst1/2 inhibitor that prevents YAP phosphorylation and stimulates its nuclear translocation and activity (40). Compared with mice in LPS alone groups, mice in LPS + XMU-MP-1 groups showed a significantly increased nuclear content of YAP (Fig. 5A), increased lung mRNA expression of CTGF and CYR61 (Fig. 5B and C), and increased lung CTGF protein expression (Fig. 5D) at 24, 48 and $72 \mathrm{~h}$ post 

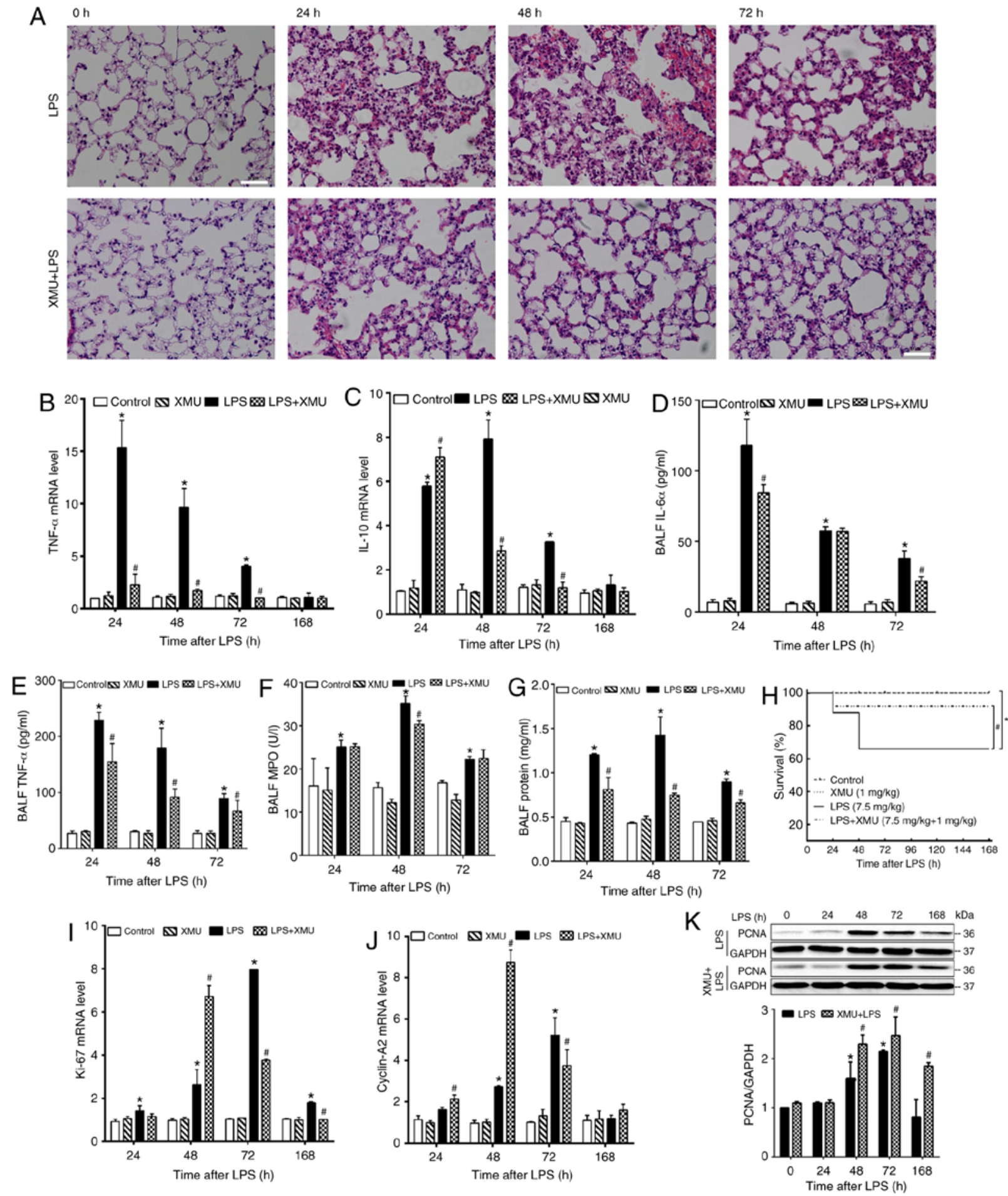

Figure 6. Stimulation of YAP activity attenuates lung injury and promotes lung recovery. Mice were intraperitoneally injected with $0.1 \%$ citric acid containing $20 \%$ Kolliphor HS (solvent for XMU-MP-1), $1 \mathrm{mg} / \mathrm{kg}$ XMU-MP-1, LPS or LPS + XMU-MP-1. The effects of stimulating YAP activity on lung injury and recovery, and on lung inflammation were evaluated at injury and repair phases. (A) Histological examination of lung injury. Similar result were obtained in all 5 mice at each respective time-point. Magnification, x200. Reverse transcription-quantitative PCR analysis of tissue mRNA expression levels of (B) TNF- $\alpha$ and (C) IL-10. Data are presented as the mean \pm SEM ( $n=3$ mice/group; 2 experimental repeats/animal). Analysis of BALF levels of (D) IL-6 $\alpha$ and (E) TNF- $\alpha$, (F) MPO activity and (G) total protein concentration. Data are presented as the mean \pm SEM ( $n=5$ mice/group). (H) Stimulation of YAP activity improved the survival rate. Analyses of tissue expression levels of (I) Ki-67 and (J) cyclin-A2 mRNA, and (K) PCNA protein. Data are presented as the mean \pm SEM (n=3 mice/group; 2 experimental repeats/animal). ${ }^{*} \mathrm{P}<0.05$ vs. the control group; and ${ }^{~} \mathrm{P}<0.05$ vs. the LPS alone group. LPS, lipopolysaccharide; XMU, XMU-MP-1; BALF, bronchoalveolar lavage fluid; PCNA, proliferating cell nuclear antigen.

LPS injection. Thus, these results confirmed that XMU-MP-1 significantly stimulated YAP activity in the lungs.

The effects of stimulating YAP activity on lung injury, recovery and inflammation were subsequently studied.
Compared with mice injected with LPS alone, mice treated with LPS + XMU-MP-1 showed a markedly attenuated tissue injury (Fig. 6A), lower tissue expression levels of TNF- $\alpha$ and Il-10 (Fig. 6B and C), lower BALF levels of IL-6 $\alpha$ and 
TNF- $\alpha$ (Fig. 6D and E), lower BALF MPO activity (Fig. 6F), and lower BALF protein content (Fig. 6G) as an indicator for lower epithelial permeability (3), at 24, 48 and $72 \mathrm{~h}$ post-LPS injection. Alleviation of lung inflammation and injury by XMU-MP-1 treatment led to an improved outcome. Compared with mice injected with LPS alone, mice treated with LPS + XMU-MP-1 showed an earlier return of tissue inflammatory cytokine expression to the control level (Fig. 6B and C), accelerated recovery from lung injury (Fig. 6A and D-G) and a higher survival rate (Fig. 6H).

Stimulation of YAP activity with XMU-MP-1 had no effect on tissue expression levels of the cell proliferation markers, including Ki-67, cyclin-A2 and PCNA (Fig. 6I-K) at $24 \mathrm{~h}$ (progressive injury phase), but the expression levels of these markers were significantly increased at $48 \mathrm{~h}$ post LPS injection (transition phase) (Fig. 6I-K). XMU-MP-1 had a varied effect on tissue expression levels of these cell proliferation markers at $72 \mathrm{~h}$ post LPS injection (active repair phase), augmenting the expression of PCNA (Fig. 6K), but inhibiting that of Ki-67 and cyclin-A2 (Fig. 6I and J).

Inhibition of YAP activity suppresses epithelial cell regeneration. Epithelial regeneration is an essential component of lung repair $(2,3,34,35)$. Using tissue protein expression levels of alveolar type I epithelial cell (AECI)-specific markers, PDPN and AQP-5 (45), and AECII specific marker, Sp-c (46), as indicators, the present study estimated the effects of LPS on the abundance of AECI and AECII cells in the lungs and assessed the effects of inhibiting the YAP activity on LPS-induced changes in AECI and AECII cell abundance. Lung tissue mRNA expression levels of PDPN and $\mathrm{Sp}-\mathrm{c}$ in the LPS group decreased significantly at 24 and $48 \mathrm{~h}$ (injury phase) compared with that in the control group, but partially returned to control levels at 72 and $168 \mathrm{~h}$ post LPS injection (repair phase) (Fig. 7A and B). Lung tissue protein expression levels of Sp-c and AQP-5 in the LPS group followed a similar pattern to the aforementioned mRNA expression levels (Fig. 7C and D). Inhibition of YAP activity with VP inhibited LPS-induced changes in PDPN mRNA expression at 24, 48, 72 and $168 \mathrm{~h}$, and in Sp-c mRNA expression at 24,72 and $168 \mathrm{~h}$ post-LPS injection (Fig. 7A and B). Inhibition of YAP activity with VP had no effects on LPS-induced downregulation of Sp-c and AQP-5 protein expression at 24 and $48 \mathrm{~h}$ (Fig. 7C and D), but significantly inhibited LPS-induced upregulation of Sp-c protein expression at 72 and $168 \mathrm{~h}$, and AQP-5 protein expression at $72 \mathrm{~h}$ post-LPS injection (Fig. 7C and D). This result is consistent with the hypothesis that lung injury is associated with loss of AECI and AECII cells, and lung repair is associated with an increased number of these cells in the lungs $(3,34,35)$. Inhibition of YAP activity impedes the restoration of AECI and AECII cell numbers in the lungs. Suppression of Hippo-YAP activity appeared to impede AECI and AECII proliferation. Compared with the numbers observed in the LPS group, lung sections from mice treated with LPS + VP had markedly lower numbers of AQP-5/Ki-67 or PDPN/Ki-67 double-positive proliferating AECI cells, and $\mathrm{Sp}-\mathrm{c} / \mathrm{Ki}-67$ positive proliferating AECII cells associated with a reduced number of Sp-c/YAP double-positive cells at 48 and $72 \mathrm{~h}$ post-LPS injection (Fig. 7E).
YAP regulates epithelial adherens junction complex formation. The increase in epithelial permeability is a hallmark of lung injury (3,34-36). To clarify whether YAP activity regulated epithelial adherens junction assembly and disassembly, the present study examined the effects of inhibiting YAP activity on the expression and intracellular translocation of membrane-bound E-cadherin, two mechanisms that regulate the assembly and disassembly of the epithelial adherens junction complex $(34.35,37)$. In the LPS group, E-cadherin mRNA expression level was significantly downregulated in the lungs at the injury phase compared with the control group, and returned to the control level in the lungs at the repair phase (Fig. 8A). Inhibition of YAP activity with VP exacerbated LPS-induced downregulation of E-cadherin expression at the injury phase and inhibited E-cadherin expression at the repair phase (Fig. 8A). In the LPS group, E-cadherin protein content was reduced in the membrane fraction and increased in the cytoplasmic fraction from the same lungs at the injury phase (Fig. 8B and C). This shift indicates an increased subcellular translocation of E-cadherin protein from the membrane to the cytoplasmic compartment in the lungs at the injury phase. Compared with control lungs, E-cadherin protein content was not altered by LPS in both membrane and cytoplasm fractions in the lungs at the repair phase (Fig. 8B and C), indicating that there was little E-cadherin internalization at this phase. Inhibition of YAP activity by VP at $48 \mathrm{~h}$ post LPS injection augmented LPS-induced E-cadherin protein decrease in the membrane fraction and resulted in an increase in the cytoplasmic fraction compared with the LPS group (Fig. 8B and C). Inhibition of YAP activity significantly decreased membrane E-cadherin levels and increased cytoplasmic E-cadherin levels in the lungs at $72 \mathrm{~h}$ post-LPS injection (Fig. 8B and C). These results indicated that YAP inhibition potentiated LPS-induced E-cadherin internalization at the injury phase and stimulated E-cadherin internalization at the repair phase.

\section{Discussion}

This present study examined the roles of the Hippo-YAP signaling pathway in lung injury, repair and inflammation. The results demonstrated that lung injury was associated with an increase in YAP phosphorylation (reduced YAP activity) and lung repair was associated with a decrease in YAP phosphorylation (increased YAP activity). Suppression of YAP activity by inhibiting the formation of the YAP-TEAD complex in the nucleus exacerbated lung inflammation, augmented the increase in epithelial permeability and worsened lung injury. Inhibition of YAP activity delayed lung inflammation resolution and recovery from injury and reduced the survival rate of mice. Stimulation of YAP activity by inhibiting Mst1/2 attenuated lung inflammation and injury, and reduced epithelial permeability. Stimulation of YAP activity also promoted lung inflammation resolution, accelerated lung recovery from injury and increased the survival rate of mice. The Hippo-YAP activity mediated cell proliferation, stimulated epithelial cell regeneration and the formation of epithelial adherens junctions, and improved lung repair. Collectively, the present study demonstrated that the YAP activity was an endogenous mechanism that protected against endotoxemic lung injury. 

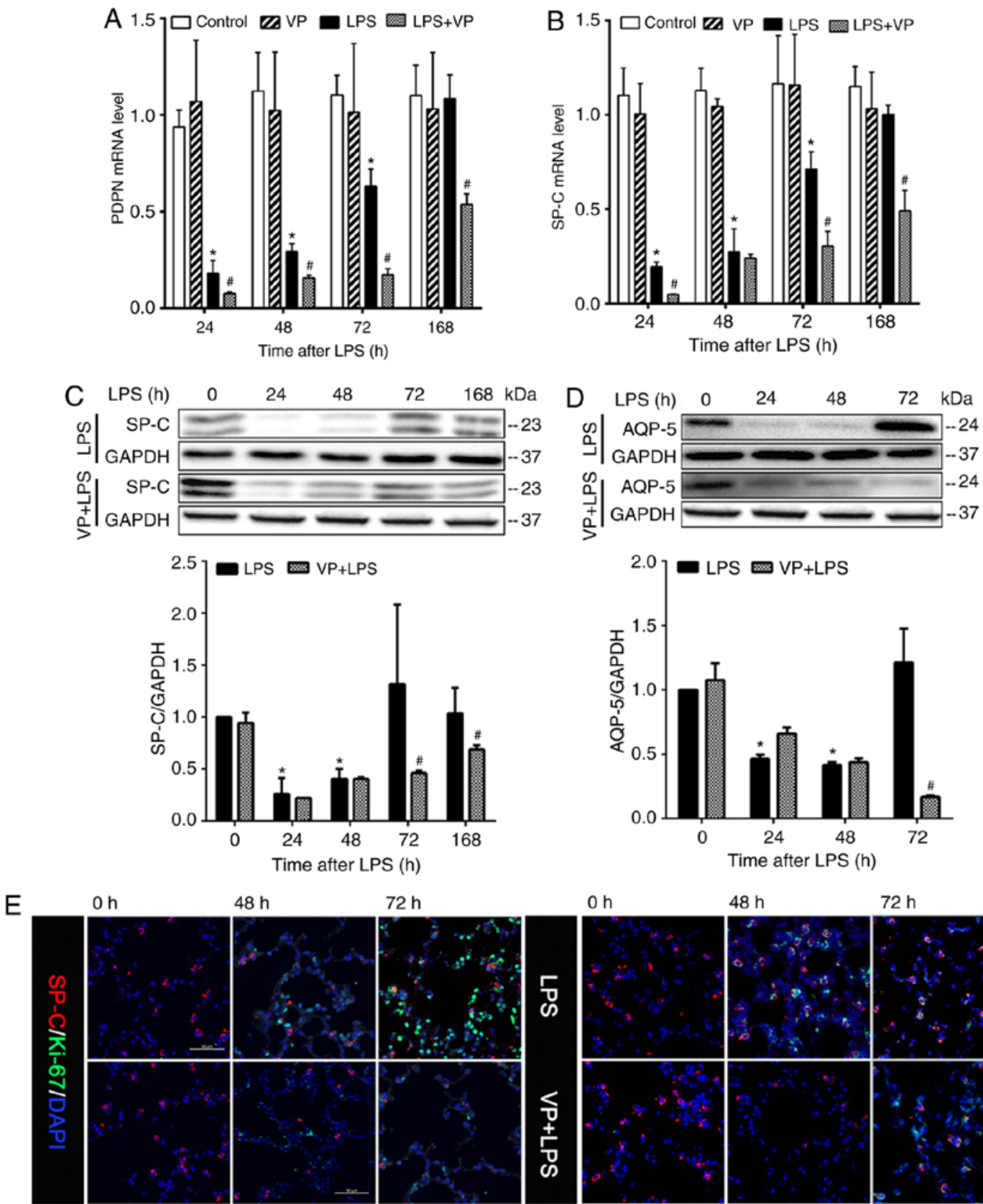

$48 \mathrm{~h}$

$72 \mathrm{~h}$
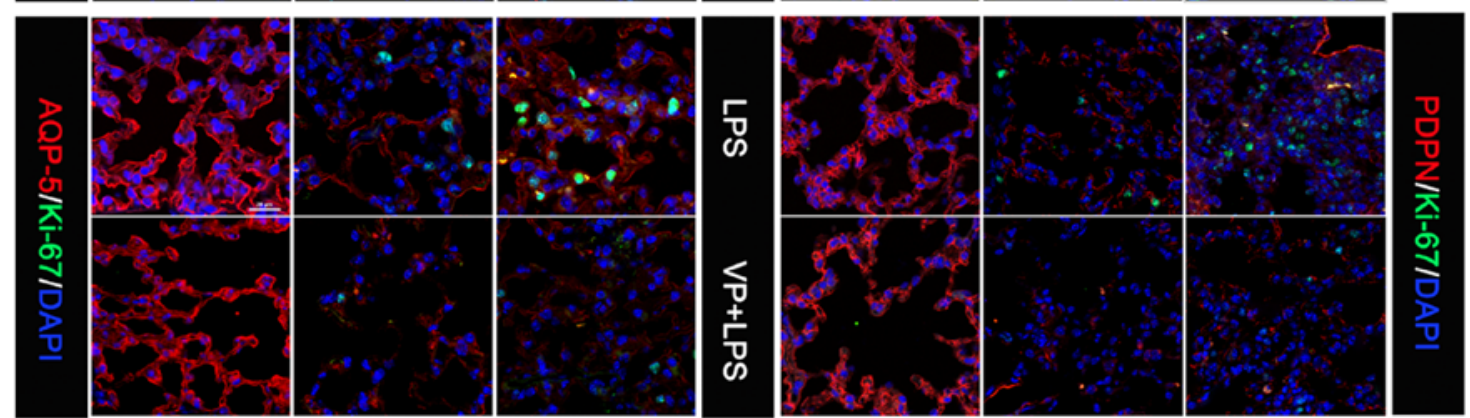

Figure 7. Inhibition of YAP activity suppresses epithelial cell regeneration. Mice were intraperitoneally injected with DMSO (solvent for VP), VP (100 mg/kg), LPS or LPS + VP. The effects of inhibiting YAP activity on lung epithelial damage and regeneration were estimated by measuring tissue levels of alveolar type I epithelial cell-specific markers, PDPN and AQP-5, and alveolar type II epithelial cell-specific marker, Sp-c, and by immunofluorescence staining of lung sections. Analysis of tissue expression levels of (A) PDPN and (B) Sp-c mRNA, and (C) AQP-5 and (D) Sp-c protein. Data are presented as the mean \pm SEM (n=3 mice/group; 2 experimental repeats/animal). ${ }^{*} \mathrm{P}<0.05$ vs. the control group; and ${ }^{\#} \mathrm{P}<0.05$ vs. the LPS alone group. (E) Immunofluorescence staining of lung sections. PDPN, podoplanin; AQP-5, aquaporin-5; Sp-c, pulmonary surfactant apoprotein C; LPS, lipopolysaccharide; VP, verteporfin; YAP, Yes-associated protein.

The Hippo-YAP activity appeared to protect against endotoxemic lung injury by different mechanisms at different pathological stages. Lung injury was associated with a marked increase in lung inflammation and disassembly of epithelial 


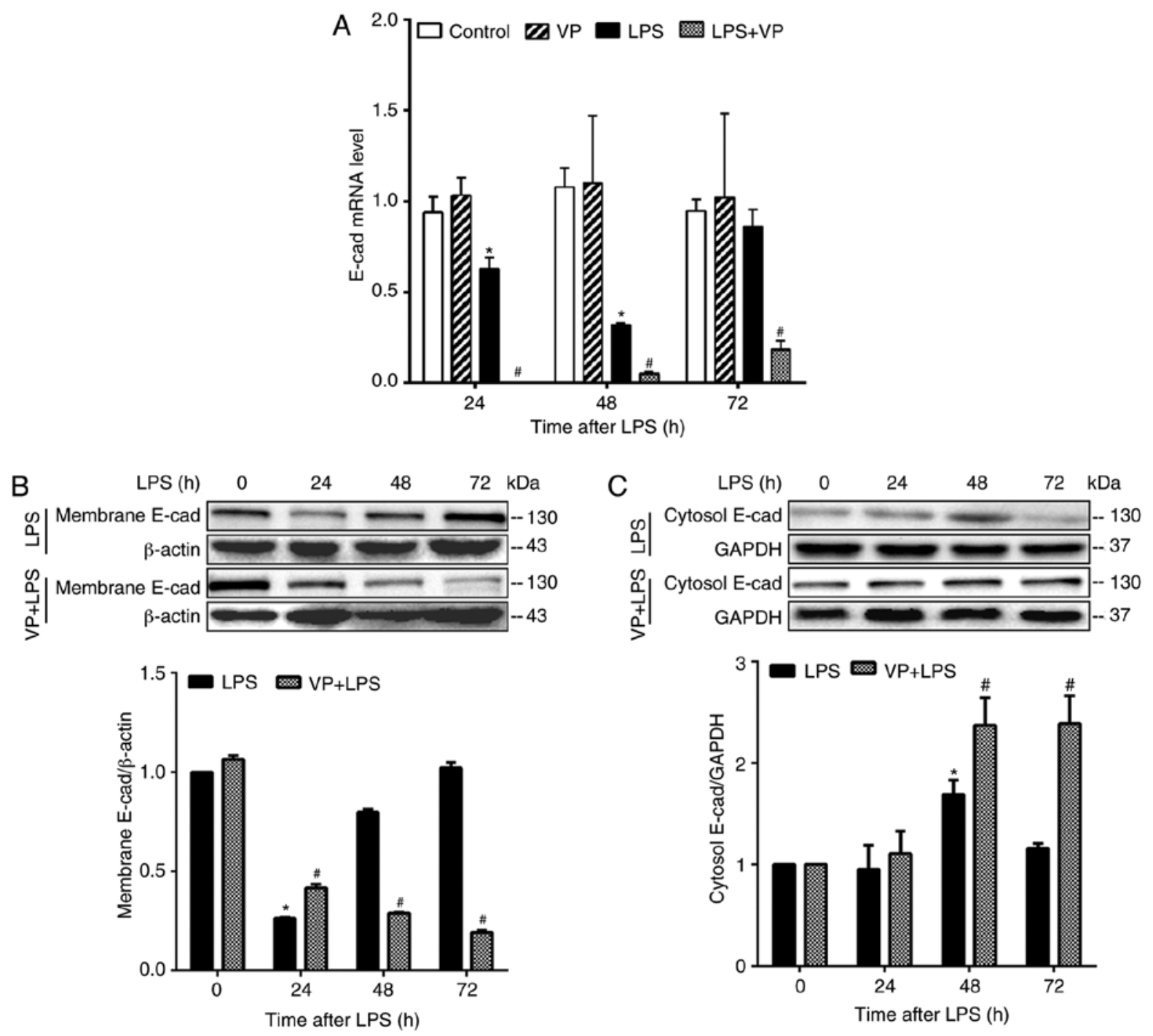

Figure 8. Inhibition of YAP activity impedes epithelial adherens junction formation. Mice were intraperitoneally injected with DMSO, VP, LPS or LPS + VP. (A) Reverse transcription-quantitative PCR analysis of E-cadherin mRNA expression. Western blot analysis of (B) membrane and (C) cytosolic E-cadherin levels. Data are presented as the mean \pm SEM ( $n=3$ mice/group). " $\mathrm{P}<0.05$ vs. the control group; and ${ }^{\text {" }} \mathrm{P}<0.05$ vs. the LPS alone group. LPS, lipopolysaccharide; VP, verteporfin; E-cad, E-cadherin.

adherens junctions. However, it was not associated with cell proliferation, epithelial cell regeneration or inflammation resolution. Inhibition of YAP activity at the injury phase exacerbated, whereas stimulation of YAP activity alleviated lung injury, reduced lung inflammation and restored the disassembled lung epithelial adherens junctions. Inhibition or stimulation of YAP activity at the injury phase had no effect on cell proliferation, epithelial cell regeneration or inflammation resolution. It is likely that Hippo-YAP activity at the injury phase protects against lung injury by inhibiting lung inflammation and preventing epithelial adherens junction disassembly. By contrast, lung repair was associated with a low level of lung inflammation, as well as increased cell proliferation, epithelial regeneration, inflammation resolution and reassembly of epithelial adherens junctions. Stimulation of YAP activity at the repair phase attenuated lung injury and inflammation, and accelerated inflammation resolution and lung recovery from injury. Inhibition of YAP activity at the repair phase exacerbated lung injury and inflammation, inhibited cell proliferation and epithelial regeneration, delayed inflammation resolution and lung recovery from injury, and impeded reassembly of epithelial adherens junctions. It is likely that at the repair phase, YAP activity protects against lung injury by stimulating cell proliferation and epithelial cell regeneration, promoting epithelial adherens junction reassembly, and accelerating lung inflammation resolution and lung recovery from injury. Thus, YAP activity may protect against lung injury by multiple mechanisms. It inhibited inflammation at the injury phase and promoted lung repair and inflammation resolution at the repair phase.

The present study demonstrated that suppression of YAP activity downregulated E-cadherin protein expression and stimulated its translocation from the membrane to the cytoplasm, suggesting that YAP activity upregulated E-cadherin expression and inhibited its internalization. E-cadherin internalization and downregulation are important mechanisms of epithelial adherens junction disassembly during lung injury, whereas translocation of E-cadherin from the cytoplasm to the membrane and its upregulation are major mechanisms of epithelial adherens junction reassembly during lung 
repair $(35,36,47,48)$. The results of the present study thus imply that YAP activity promoted epithelial adherens complex assembly and adherens junction formation, and thereby epithelial barrier repair. Collectively, YAP activity mediated both epithelial proliferation (new cell generation) and epithelial cell-cell junction formation, two key steps in epithelial barrier repair and restoration $(35,36,38,47,48)$. YAP/TAZ activity has been shown to stimulate endothelial proliferation and increase VE-cadherin turnover at inter-endothelial junctions to maintain endothelial junction stability and barrier function $(49,50)$. Thus, it may be hypothesized that the Hippo-YAP signaling pathway simultaneously promotes new epithelial/endothelial cell generation and stimulates cell-cell junction formation and stability. The Hippo-YAP signaling pathway might be playing a balancing role in regulating epithelial/endothelial barrier repair following inflammatory lung injury.

The findings of the present study may have therapeutic implications. The steps involved in the epithelial/endothelial barrier repair process remain to be elucidated, but are likely to involve three phases $(34,35,37,38)$. First, the generation of new epithelial or endothelial cells (EpiCs or ECs) through the division of living EpiCs or ECs adjacent to damaged or dead cells, or through differentiation of other epithelial or endothelial precursor cells. Second, integration of the newly generated EpiCs or ECs into the epithelial or endothelial layer to replace the damaged or dead EpiCs or ECs. Third, formation of stable cell-cell junctions between the new EpiCs or ECs and the neighboring EpiCs or ECs in the epithelium or endothelium to restore normal epithelial or endothelial barrier function $(34,35,37,38)$. Formation of intercellular junctions between the new EpiCs or ECs and neighboring EpiCs or ECs is crucial for epithelial or endothelial barrier repair, although cell division/proliferation to generate new EpiCs or ECs is also necessary $(34,35,37,38)$. To facilitate epithelial or endothelial barrier repair, cell division/proliferation and intercellular junction formation must be regulated in a coordinated manner. Numerous pathways have been shown to stimulate cell division/proliferation $(34,35)$. However, few of them also stimulate the formation of intercellular junctions. Mechanisms and pathways that stimulate EpiC or EC division/proliferation, but do not stimulate the formation of intercellular junctions would impede rather than promote epithelial or endothelial barrier repair. Mitotic cell rounding and cytokinesis during cell division cause extensive rearrangement and reconstruction of epithelial or endothelial adherens and tight junctions (51). This creates new leaky sites in the epithelial or endothelial layer by opening new paracellular pathways. The more EpiCs or ECs undergo cell division, the more new leaky sites are created. This impedes rather than promotes epithelial or endothelial barrier repair. Thus, the identification of mechanisms and pathways that promote both cell proliferation and cell-cell junction formation is valuable for lung regeneration. The results of the present study indicated that the Hippo-YAP signaling pathway stimulated EpiC proliferation and epithelial adherens junction complex formation suggesting that stimulating YAP activity could be a useful strategy to promote epithelial barrier repair.

Previous studies demonstrated that selective blockade of endothelial or type II epithelial YAP activity exacerbates endothelial and lung inflammation (52) or impedes lung inflammation resolution (32). The current findings that the Hippo-YAP signaling pathway inhibits inflammation and promotes inflammation resolution in the lungs are consistent with these reports. The results of the present study are also in line with a previous study which reported that epicardial YAP/TAZ regulated an immunosuppressive response following myocardial infarction (53). However, the current findings are inconsistent with another study, in which it was demonstrated that endothelial-specific YAP overexpression exacerbates, whereas selective YAP knockdown in the endothelium inhibits vascular wall inflammation (54). To the best of our knowledge, the present report is the first to examine the effects of systemic stimulation and inhibition of YAP activity on lung inflammation, injury and repair. The present study extrapolates those prior studies by demonstrating that YAP activity protects against lung injury by activating different mechanisms at different pathological stages and that it stimulates both EpiC proliferation and epithelial adherens junction formation.

In conclusion, the results of the present study demonstrated that YAP activity is an endogenous protective mechanism against endotoxemic lung injury. YAP activity appears to protect against lung injury by activating different mechanisms at different pathological stages. At the injury phase, YAP activity protected against lung injury by inhibiting lung inflammation and preventing epithelial adherens junction disassembly. At the repair phase, it promoted lung recovery by stimulating cell proliferation and epithelial cell regeneration, promoting epithelial adherens junction reassembly, and accelerating lung inflammation resolution. Thus, YAP activity protects against lung injury by multiple mechanisms. Stimulation of EpiC proliferation and epithelial adherens junction formation suggests that stimulating YAP activity could be a useful strategy to promote epithelial barrier repair.

Although the present study demonstrated that YAP activity protected against lung injury by acting at injury and repair phases and by activating multiple mechanisms, the therapeutic effects of stimulating YAP activity on lung injury were not examined, and will be the focus of a future study. Mice will be injected with VP and LPS or XMU-MP-1 and LPS simultaneously, or administered VP or XMU-MP-1 at 6, 12 or $24 \mathrm{~h}$ after LPS injection. The effects of these interventions on LPS-induced lung inflammation and injury, on lung repair and inflammation resolution, and on overall outcomes will be assessed. These studies will allow for evaluation of the therapeutic value of stimulating YAP activity for the treatment of ALI.

\section{Acknowledgements}

Not applicable.

\section{Funding}

This work was supported by the Natural Science Foundation of Zhejiang Province of China (grant nos. LY18H010007 and LY17H010007) and the Natural Science Foundation of China (grant no. 81370171). 


\section{Availability of data and materials}

The datasets used and/or analyzed during the current study are available from the corresponding author on reasonable request.

\section{Authors' contributions}

YSG, SZM and SFL contributed to the study conception and design, supervision of all experiments, and acquisition of funding. LYL, XQS, FKZ, XFF, YYW and JMF contributed to the study design, and participated in data acquisition, analysis and interpretation. All authors have read and approved the final manuscript.

\section{Ethics approval and consent to participate}

All animal experiments were approved by the Institutional Animal Care and Use Committee of Wenzhou Medical University (Wenzhou, China) and were performed in accordance with the Guide for the Care and Use of Laboratory Animals of the National Institute of Health.

\section{Patient consent for publication}

Not applicable.

\section{Competing interests}

The authors declare that they have no competing interests.

\section{References}

1. Ware LB and Matthay MA: The acute respiratory distress syndrome. N Engl J Med 342: 1334-1349, 2000.

2. Matthay MA and Zimmerman GA: Acute lung injury and the acute respiratory distress syndrome: Four decades of inquiry into pathogenesis and rational management. Am J Respir Cell Mol Biol 33: 319-327, 2005.

3. Matthay MA, Ware LB and Zimmerman GA: The acute respiratory distress syndrome. J Clin Invest 122: 2731-2740, 2012.

4. Li G, Malinchoc M, Cartin-Ceba R, Venkata CV, Kor DJ, Peters SG, Hubmayr RD and Gajic O: Eight-year trend of acute respiratory distress syndrome: A population-based study in Olmsted County, Minnesota. Am J Respir Crit Care Med 183: 59-66, 2011.

5. Rubenfeld GD, Caldwell E, Peabody E, Weaver J, Martin DP Neff M, Stern EJ and Hudson LD: Incidence and outcomes of acute lung injury. N Engl J Med 353: 1685-1693, 2005.

6. Tsushima K, King LS, Aggarwal NR, De Gorodo A, D'Alessio FR and Kubo K: Acute lung injury review. Intern Med 48: 621-630, 2009.

7. Moya IM and Halder G: Hippo-YAP/TAZ signalling in organ regeneration and regenerative medicine. Nat Rev Mol Cell Biol 20: 211-226, 2019.

8. Zhao B, Tumaneng K and Guan KL: The Hippo pathway in organ size control, tissue regeneration and stem cell self-renewal. Nat Cell Biol 13: 877-883, 2011.

9. Dong J, Feldmann G, Huang J, Wu S, Zhang N, Comerford SA, Gayyed MF, Anders RA, Maitra A and Pan D: Elucidation of a universal size-control mechanism in Drosophila and mammals. Cell 130: 1120-1133, 2007.

10. Xie H, Wu L, Deng Z, Huo Y and Cheng Y: Emerging roles of YAP/TAZ in lung physiology and diseases. Life Sci 214: 176-183, 2018.

11. Xin M, Kim Y, Sutherland LB, Murakami M, Qi X, McAnally J, Porrello ER, Mahmoud AI, Tan W, Shelton JM, et al: Hippo pathway effector Yap promotes cardiac regeneration. Proc Natl Acad Sci USA 110: 13839-13844, 2013.
12. Wu S, Liu Y, Zheng Y, Dong J and Pan D: The TEAD/TEF family protein Scalloped mediates transcriptional output of the Hippo growth-regulatory pathway. Dev Cell 14: 388-398, 2008.

13. Wang X, Freire Valls A, Schermann G, Shen Y, Moya IM, Castro L, Urban S, Solecki GM, Winkler F, Riedemann L, et al: YAP/TAZ Orchestrate VEGF signaling during developmental angiogenesis. Dev Cell 42: 462-478.e7, 2017.

14. Elaimy AL and Mercurio AM: Convergence of VEGF and YAP/TAZ signaling: Implications for angiogenesis and cancer biology. Sci Signal 11: eaau1165, 2018.

15. Piccolo S, Dupont S and Cordenonsi M: The biology of YAP/TAZ: Hippo signaling and beyond. Physiol Rev 94: 1287-1312, 2014.

16. Gumbiner BM and Kim NG: The Hippo-YAP signaling pathway and contact inhibition of growth. J Cell Sci 127: 709-717, 2014.

17. Saito A and Nagase T: Hippo and TGF- $\beta$ interplay in the lung field. Am J Physiol Lung Cell Mol Physiol 309: L756-L767, 2015.

18. Zhou B, Flodby P, Luo J, Castillo DR, Liu Y, Yu FX, McConnell A, Varghese B, Li G, Chimge NO, et al: Claudin-18-mediated YAP activity regulates lung stem and progenitor cell homeostasis and tumorigenesis. J Clin Invest 128: 970-984, 2018.

19. Lange AW, Sridharan A, Xu Y, Stripp BR, Perl AK and Whitsett JA: Hippo/Yap signaling controls epithelial progenitor cell proliferation and differentiation in the embryonic and adult lung. J Mol Cell Biol 7: 35-47, 2015.

20. Volckaert T, Yuan T, Yuan J, Boateng E, Hopkins S, Zhang JS, Thannickal VJ, Fässler R and De Langhe SP: Hippo signaling promotes lung epithelial lineage commitment by curbing Fgf 10 and $\beta$-catenin signaling. Development 146: dev166454, 2019.

21. Zhao R, Fallon TR, Saladi SV, Pardo-Saganta A, Villoria J, Mou H, Vinarsky V, Gonzalez-Celeiro M, Nunna N, Hariri LP, et al: Yap tunes airway epithelial size and architecture by regulating the identity, maintenance, and self-renewal of stem cells. Dev Cell 30: 151-165, 2014.

22. Azad T, Ghahremani M and Yang X: The role of YAP and TAZ in angiogenesis and vascular mimicry. Cells 8: 407, 2019.

23. Choi HJ, Zhang H, Park H, Choi KS, Lee HW, Agrawal V, Kim YM and Kwon YG: Yes-associated protein regulates endothelial cell contact-mediated expression of angiopoietin-2. Nat Commun 6: 6943, 2015 .

24. Sharif GM, Schmidt MO, Yi C, Hu Z, Haddad BR, Glasgow E, Riegel AT and Wellstein A: Cell growth density modulates cancer cell vascular invasion via Hippo pathway activity and CXCR2 signaling. Oncogene 34: 5879-5889, 2015.

25. Zhao B, Li L, Wang L, Wang CY, Yu J and Guan KL: Cell detachment activates the Hippo pathway via cytoskeleton reorganization to induce anoikis. Genes Dev 26: 54-68, 2012.

26. Liu F, Lagares D, Choi KM, Stopfer L, Marinković A, Vrbanac V, Probst CK, Hiemer SE, Sisson TH, Horowitz JC, et al: Mechanosignaling through YAP and TAZ drives fibroblast activation and fibrosis. Am J Physiol Lung Cell Mol Physiol 308: L344-L357, 2015.

27. Chung C, Kim T, Kim M, Kim M, Song H, Kim TS, Seo E, Lee SH, Kim H, Kim SK, et al: Hippo-Foxa2 signaling pathway plays a role in peripheral lung maturation and surfactant homeostasis. Proc Natl Acad Sci USA 110: 7732-7737, 2013.

28. Mindos T, Dun XP, North K, Doddrell RD, Schulz A, Edwards P, Russell J, Gray B, Roberts SL, Shivane A, et al: Merlin controls the repair capacity of Schwann cells after injury by regulating Hippo/YAP activity. J Cell Biol 216: 495-510, 2017.

29. Liu Y, Lu T, Zhang C, Xu J, Xue Z, Busuttil RW, Xu N, Xia Q, Kupiec-Weglinski JW and Ji H: Activation of YAP attenuates hepatic damage and fibrosis in liver ischemia-reperfusion injury. J Hepatol 71: 719-730, 2019.

30. Li L, Dong L, Zhang J, Gao F, Hui J and Yan J: Mesenchymal stem cells with downregulated Hippo signaling attenuate lung injury in mice with lipopolysaccharide-induced acute respiratory distress syndrome. Int J Mol Med 43: 1241-1252, 2019.

31. Volckaert T, Yuan T, Chao CM, Bell H, Sitaula A, Szimmtenings L, El Agha E, Chanda D, Majka S, Bellusci S, et al: Fgf10-Hippo epithelial-mesenchymal crosstalk maintains and recruits lung basal stem cells. Dev Cell 43: 48-59.e5, 2017.

32. LaCanna R, Liccardo D, Zhang P, Tragesser L, Wang Y, Cao T, Chapman HA, Morrisey EE, Shen H, Koch WJ, et al: Yap/Taz regulate alveolar regeneration and resolution of lung inflammation. J Clin Invest 129: 2107-2122, 2019.

33. Hu C, Sun J, Du J, Wen D, Lu H, Zhang H, Xue Y, Zhang A, Yang $\mathrm{C}$, Zeng $\mathrm{L}$ and Jiang $\mathrm{J}$ : The Hippo-YAP pathway regulates the proliferation of alveolar epithelial progenitors after acute lung injury. Cell Biol Int 43: 1174-1183, 2019. 
34. Bhattacharya $\mathbf{J}$ and Matthay MA: Regulation and repair of the alveolar-capillary barrier in acute lung injury. Annu Rev Physiol 75: 593-615, 2013.

35. Crosby LM and Waters CM: Epithelial repair mechanisms in the lung. Am J Physiol Lung Cell Mol Physiol 298: L715-L731, 2010.

36. Frank JA: Claudins and alveolar epithelial barrier function in the lung. Ann N Y Acad Sci 1257: 175-183, 2012.

37. Liu G, Ye X, Miller EJ and Liu SF: NF-кB-to-AP-1 switch: A mechanism regulating transition from endothelial barrier injury to repair in endotoxemic mice. Sci Rep 4: 5543, 2014.

38. Mao SZ, Ye X, Liu G, Song D and Liu SF: Resident endothelial cells and endothelial progenitor cells restore endothelial barrier function after inflammatory lung injury. Arterioscler Thromb Vasc Biol 35: 1635-1644, 2015.

39. National Research Council (US) Committee for the Update of the Guide for the Care and Use of Laboratory Animals: Guide for the Care and Use of Laboratory Animals. 8th edition. National Academies Press (US), Washington, DC, 2011.

40. Fan F, He Z, Kong LL, Chen Q, Yuan Q, Zhang S, Ye J, Liu H, Sun X, Geng J, et al: Pharmacological targeting of kinases MST1 and MST2 augments tissue repair and regeneration. Sci Transl Med 8: 352ra108, 2016.

41. Livak KJ and Schmittgen TD: Analysis of relative gene expression data using real-time quantitative PCR and the 2(-Delta Delta C(T)) method. Methods 25: 402-408, 2001.

42. Ye X, Ding J, Zhou X, Chen G and Liu SF: Divergent roles of endothelial NF-kappaB in multiple organ injury and bacterial clearance in mouse models of sepsis. J Exp Med 205: 1303-1315 2008.

43. Liu SF, Ye $X$ and Malik AB: In vivo inhibition of nuclear factor-kappa $B$ activation prevents inducible nitric oxide synthase expression and systemic hypotension in a rat model of septic shock. J Immunol 159: 3976-3983, 1997.

44. Liu-Chittenden Y, Huang B, Shim JS, Chen Q, Lee SJ, Anders RA, Liu JO and Pan D: Genetic and pharmacological disruption of the TEAD-YAP complex suppresses the oncogenic activity of YAP. Genes Dev 26: 1300-1305, 2012.
45. Williams MC: Alveolar type I cells: Molecular phenotype and development. Annu Rev Physiol 65: 669-695, 2003.

46. Rippon HJ, Ali NN, Polak JM and Bishop AE: Initial observations on the effect of medium composition on the differentiation of murine embryonic stem cells to alveolar type II cells. Cloning Stem Cells 6: 49-56, 2004.

47. Bruser L and Bogdan S: Adherens junctions on the move-membrane trafficking of E-cadherin. Cold Spring Harb Perspect Biol 9: a029140, 2017.

48. Rezaee F and Georas SN: Breaking barriers. New insights into airway epithelial barrier function in health and disease. Am J Respir Cell Mol Biol 50: 857-869, 2014.

49. Kim J, Kim YH, Kim J, Park DY, Bae H, Lee DH, Kim KH, Hong SP, Jang SP, Kubota Y, et al: YAP/TAZ regulates sprouting angiogenesis and vascular barrier maturation. J Clin Invest 127: 3441-3461, 2017.

50. Neto F, Klaus-Bergmann A, Ong YT, Alt S, Vion AC, Szymborska A, Carvalho JR, Hollfinger I, Bartels-Klein E, Franco CA, et al: YAP and TAZ regulate adherens junction dynamics and endothelial cell distribution during vascular development. Elife 7: e31037, 2018.

51. Harris TJ and Tepass U: Adherens junctions: From molecules to morphogenesis. Nat Rev Mol Cell Biol 11: 502-514, 2010

52. Lv Y, Kim K, Sheng Y, Cho J, Qian Z, Zhao YY, Hu G, Pan D, Malik AB and Hu G: YAP controls endothelial activation and vascular inflammation through TRAF6. Circ Res 123: 43-56, 2018.

53. Ramjee V, Li D, Manderfield LJ, Liu F, Engleka KA, Aghajanian H, Rodell CB, Lu W, Ho V, Wang T, et al: Epicardial YAP/TAZ orchestrate an immunosuppressive response following myocardial infarction. J Clin Invest 127: 899-911, 2017.

54. Wang L, Luo JY, Li B, Tian XY, Chen LJ, Huang Y, Liu J, Deng D, Lau CW, Wan S, et al: Integrin-YAP/TAZ-JNK cascade mediates atheroprotective effect of unidirectional shear flow. Nature 540: 579-582, 2016. 